\title{
Chemical Reaction Barrier Heights by Multiconfiguration Pair-Density Functional Theory with Correlated Participating Orbitals
}

\author{
Andrew M. Sand,$\uparrow$ Katherine M. Kidder, $^{\dagger}$ Donald G. Truhlar, ${ }^{\dagger}$ and Laura Gagliardi ${ }^{\dagger}$ \\ $\dagger$ Department of Chemistry, Chemical Theory Center, and the Minnesota Supercomputing Institute, \\ The University of Minnesota, Minneapolis, MN 55455 USA \\ † Department of Chemistry and Biochemistry, Butler University, Indianapolis, IN 46208 USA
}

Table S1 - Mean signed errors (MSE) and mean unsigned errors (MUE) (in kcal/mol) for the forward and reverse reactions for the hydrogen transfer reactions (R1-R3).

\begin{tabular}{|c|c|c|c|c|c|c|c|}
\hline \multirow[b]{2}{*}{ basis set } & & \multicolumn{3}{|c|}{ nom-CPO } & \multicolumn{3}{|c|}{ mod-CPO } \\
\hline & & CASSCF & CASPT2 & tPBE & CASSCF & CASPT2 & tPBE \\
\hline maug-cc-pVDZ & MSE & 11.4 & 0.4 & -1.5 & 11.2 & 1.1 & -3.2 \\
\hline maug-cc-pVTZ & MSE & 12.0 & 0.8 & -0.8 & 9.2 & 0.3 & -2.4 \\
\hline maug-cc-pVDZ & MUE & 11.4 & 1.9 & 1.7 & 11.2 & 1.4 & 3.2 \\
\hline maug-cc-pVTZ & MUE & 12.0 & 0.9 & 1.5 & 9.2 & 0.6 & 2.4 \\
\hline
\end{tabular}

Table S2 - Mean signed errors (MSE) and mean unsigned errors (MUE) (in $\mathrm{kcal} / \mathrm{mol}$ ) for the forward and reverse reactions for the rearrangement reactions ( $\mathbf{R 4}$ and $\mathbf{R 1 2})$.

\begin{tabular}{|c|c|c|c|c|c|c|c|}
\hline \multirow[b]{2}{*}{ basis set } & & \multicolumn{3}{|c|}{ nom-СРО } & \multicolumn{3}{|c|}{ mod-CРO } \\
\hline & & CASSCF & CASPT2 & tPBE & CASSCF & CASPT2 & $\mathrm{tPBE}$ \\
\hline maug-cc-pVDZ & MSE & 2.8 & -0.9 & -4.4 & 2.8 & -0.2 & -4.4 \\
\hline maug-cc-pVTZ & MSE & 3.3 & -0.3 & -4.2 & 3.3 & -0.3 & -3.7 \\
\hline maug-cc-pVDZ & MUE & 14.5 & 3.9 & 4.4 & 14.5 & 4.5 & 4.4 \\
\hline maug-cc-pVTZ & MUE & 13.8 & 3.0 & 4.2 & 13.6 & 3.0 & 4.2 \\
\hline
\end{tabular}


Table S3 - Mean signed errors (MSE) and mean unsigned errors (MUE) (in $\mathrm{kcal} / \mathrm{mol}$ ) for the forward and reverse reactions for the heavy-atom transfer reactions (R5 and R6).

\begin{tabular}{llrrrrrrr} 
& & \multicolumn{3}{c}{ nom-CPO } & & \multicolumn{3}{c}{ mod-CPO } \\
\cline { 3 - 5 } \cline { 8 - 10 } basis set & & CASSCF & CASPT2 & tPBE & & CASSCF & CASPT2 & tPBE \\
\hline maug-cc-pVDZ & MSE & 7.4 & 3.4 & -0.3 & & 13.8 & 2.2 & -5.2 \\
maug-cc-pVTZ & MSE & 9.0 & 2.5 & 0.2 & & 15.9 & 4.3 & -5.3 \\
\hline maug-cc-pVDZ & MUE & 7.4 & 5.3 & 2.2 & & 13.8 & 4.3 & 5.2 \\
maug-cc-pVTZ & MUE & 9.0 & 2.7 & -1.0 & & 15.9 & 4.6 & 5.3 \\
\hline
\end{tabular}

Table S4 - Mean signed errors (MSE) and mean unsigned errors (MUE) (in $\mathrm{kcal} / \mathrm{mol}$ ) for the forward and reverse reactions for the $\mathrm{S}_{\mathrm{N}} 2$ reactions $(\mathbf{R} 7-\mathbf{R} 9)$.

\begin{tabular}{|c|c|c|c|c|c|c|c|}
\hline \multirow[b]{2}{*}{ basis set } & & \multicolumn{3}{|c|}{ nom-CPO } & \multicolumn{3}{|c|}{ mod-CPO } \\
\hline & & CASSCF & CASPT2 & tPBE & CASSCF & CASPT2 & tPBE \\
\hline maug-cc-pVDZ & MSE & 7.2 & 1.1 & -0.7 & 3.8 & 3.3 & 2.6 \\
\hline maug-cc-pVTZ & MSE & 9.1 & -0.3 & -2.0 & 5.1 & 1.2 & -2.0 \\
\hline maug-cc-pVDZ & MUE & 7.2 & 1.9 & 1.5 & 3.8 & 3.3 & 2.7 \\
\hline maug-cc-pVTZ & MUE & 9.1 & 0.9 & 2.4 & 5.1 & 1.2 & 3.4 \\
\hline
\end{tabular}

Table S5 - Mean signed errors (MSE) and mean unsigned errors (MUE) (in $\mathrm{kcal} / \mathrm{mol}$ ) for the forward and reverse reactions for the association reactions ( $\mathbf{R 1 0}$ and $\mathbf{R 1 1})$.

\begin{tabular}{|c|c|c|c|c|c|c|c|}
\hline \multirow[b]{2}{*}{ basis set } & & \multicolumn{3}{|c|}{ nom-CPO } & \multicolumn{3}{|c|}{ mod-CPO } \\
\hline & & CASSCF & CASPT2 & tPBE & CASSCF & CASPT2 & tPBE \\
\hline maug-cc-pVDZ & MSE & 3.4 & 1.1 & 1.4 & 3.0 & 1.1 & 1.9 \\
\hline maug-cc-pVTZ & MSE & 3.4 & 0.7 & 1.4 & 3.0 & 1.8 & 1.9 \\
\hline maug-cc-pVDZ & MUE & 6.9 & 1.5 & 1.9 & 9.0 & 2.0 & 2.1 \\
\hline maug-cc-pVTZ & MUE & 7.1 & 0.9 & 1.8 & 9.2 & 1.1 & 2.0 \\
\hline
\end{tabular}


Table S6 - Absolute energies (in Hartrees) and coordinates (in Ångstroms) of each converged reactant, product, and transition state structure in the DBH24 database with both the maug-cc-pVDZ and maugcc-pVTZ basis sets.

\begin{tabular}{|c|c|c|c|}
\hline \multicolumn{4}{|c|}{$\mathbf{R} 1: \mathrm{OH}+\mathrm{CH}_{4} \rightarrow \mathrm{CH}_{3}+\mathrm{H}_{2} \mathrm{O}$} \\
\hline \multicolumn{4}{|c|}{ Reactants } \\
\hline \multicolumn{4}{|c|}{ CASSCF } \\
\hline \multicolumn{4}{|c|}{ maug-cc-pVDZ } \\
\hline \multicolumn{4}{|c|}{-115.61967612656848} \\
\hline $\mathrm{C}$ & 0.00013754 & -0.00000004 & -0.00002129 \\
\hline $\mathrm{H}$ & -0.00050317 & -0.00000055 & 1.09084130 \\
\hline $\mathrm{H}$ & 1.02882547 & 0.00000133 & -0.36304176 \\
\hline $\mathrm{H}$ & -0.51388947 & -0.89068305 & -0.36394261 \\
\hline $\mathrm{H}$ & -0.51389149 & 0.89068216 & -0.36394173 \\
\hline $\mathrm{O}$ & -23.57684685 & 0.00000005 & 9.40701454 \\
\hline $\mathrm{H}$ & -23.56428403 & 0.00000010 & 10.38776155 \\
\hline \multicolumn{4}{|c|}{ maug-cc-pVTZ } \\
\hline \multicolumn{4}{|c|}{-115.65313591435648} \\
\hline $\mathrm{C}$ & -0.00578634 & -0.00000000 & 0.00262193 \\
\hline $\mathrm{H}$ & -0.00662780 & -0.00000005 & 1.08474061 \\
\hline $\mathrm{H}$ & 1.01472530 & 0.00000004 & -0.35729225 \\
\hline $\mathrm{H}$ & -0.51562490 & -0.88354330 & -0.35847986 \\
\hline $\mathrm{H}$ & -0.51562495 & 0.88354330 & -0.35847979 \\
\hline $\mathrm{O}$ & -23.58197709 & -0.00000010 & 9.40452062 \\
\hline $\mathrm{H}$ & -23.52953623 & 0.00000010 & 10.37703873 \\
\hline
\end{tabular}

\section{CASPT2}

maug-cc-pVDZ

\begin{tabular}{cccc}
\multicolumn{5}{c}{-115.91934874290739} & \\
$\mathrm{C}$ & 0.00234006 & 0.00000000 & -0.00098788 \\
$\mathrm{H}$ & 0.00223154 & -0.00000001 & 1.09872882 \\
$\mathrm{H}$ & 1.03918578 & -0.00000003 & -0.36745921 \\
$\mathrm{H}$ & -0.51604024 & -0.89790579 & -0.36760041 \\
$\mathrm{H}$ & -0.51604018 & 0.89790583 & -0.36760039 \\
$\mathrm{O}$ & -23.57587893 & -0.00000008 & 9.40981533 \\
$\mathrm{H}$ & -23.57625003 & 0.00000008 & 10.38977374 \\
maug-cc-pVTZ & & \\
-116.03717003797134 & \\
$\mathrm{C}$ & 0.00077424 & -0.00000002 & -0.00033262 \\
$\mathrm{H}$ & 0.00059560 & -0.00000023 & 1.08509552 \\
$\mathrm{H}$ & 1.02416856 & 0.00000045 & -0.36197135 \\
$\mathrm{H}$ & -0.51084563 & -0.88623735 & -0.36221161 \\
$\mathrm{H}$ & -0.51084611 & 0.88623710 & -0.36221145 \\
$\mathrm{O}$ & -23.57216540 & -0.00000002 & 9.41241099 \\
$\mathrm{H}$ & -23.57213326 & 0.00000006 & 10.38389053
\end{tabular}




\section{tPBE}

maug-cc-pVDZ

$\begin{array}{cccc}\mathrm{C} & 0.00299272 & 0.00000001 & -0.00125656 \\ \mathrm{H} & 0.00290598 & 0.00000002 & 1.10364347 \\ \mathrm{H} & 1.04472789 & -0.00000004 & -0.36947583 \\ \mathrm{H} & -0.51784904 & -0.90214125 & -0.36958984 \\ \mathrm{H} & -0.51784910 & 0.90214125 & -0.36958979 \\ \mathrm{O} & -23.57783081 & -0.00000000 & 9.40717637 \\ \mathrm{H} & -23.57754963 & 0.00000000 & 10.39376218 \\ \text { maug-cc-pVTZ } & & \\ -116.12216405871089 & \\ \mathrm{C} & 0.00175516 & 0.00000003 & -0.00072659 \\ \mathrm{H} & 0.00213227 & 0.00000234 & 1.09358794 \\ \mathrm{H} & 1.03336201 & -0.00000543 & -0.36585260 \\ \mathrm{H} & -0.51424621 & -0.89350048 & -0.36531206 \\ \mathrm{H} & -0.51423767 & 0.89350419 & -0.36531555 \\ \mathrm{O} & -23.57487189 & -0.00000037 & 9.40973437 \\ \mathrm{H} & -23.57434567 & -0.00000027 & 10.38855449\end{array}$

Products

CASSCF

maug-cc-pVDZ

$-115.61924062137182$

C $\quad 0.00697730 \quad-0.00000317 \quad 0.00358559$

$\begin{array}{llll}\mathrm{H} & 0.00732554 & 0.00000167 & 1.08339658\end{array}$

$\mathrm{H} \quad 0.94194647 \quad 0.00000131 \quad-0.53662199$

$\mathrm{H} \quad-0.92834139 \quad 0.00000016 \quad-0.53601633$

$\begin{array}{llll}\mathrm{O} & -38.95952549 & -0.00000007 & -22.48679023\end{array}$

$\mathrm{H} \quad-39.74574648 \quad-0.00000006 \quad-21.92615847$

$\mathrm{H} \quad-39.29948194 \quad 0.00000015 \quad-23.37061616$

maug-cc-pVTZ

$-115.65470854484566$

$\begin{array}{cccl}\mathrm{C} & 0.00069152 & 0.00023625 & 0.00001429 \\ \mathrm{H} & 0.00080874 & -0.00009316 & 1.06976895 \\ \mathrm{H} & 0.92706744 & -0.00009485 & -0.53496497 \\ \mathrm{H} & -0.92580199 & -0.00004712 & -0.53476070 \\ \mathrm{O} & -38.95669671 & -0.00000045 & -22.48530223 \\ \mathrm{H} & -39.73106919 & -0.00000120 & -21.91849309 \\ \mathrm{H} & -39.29184581 & 0.00000054 & -23.36548326\end{array}$




\section{CASPT2}

maug-cc-pVDZ

$-115.93814464913837$

$\begin{array}{llll}\text { C } & 0.01499771 & 0.00000024 & 0.00812895\end{array}$

$\begin{array}{llll}\mathrm{H} & 0.01503103 & 0.00000001 & 1.09809835\end{array}$

$\mathrm{H} \quad 0.95890644 \quad 0.00000002 \quad-0.53691505$

$\mathrm{H} \quad-0.92900240 \quad-0.00000028 \quad-0.53683109$

$\begin{array}{llll}\mathrm{O} & -38.96330394 & 0.00000002 & -22.48155667\end{array}$

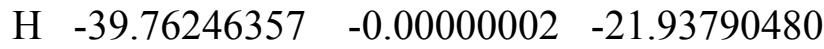

$\begin{array}{llll}\mathrm{H} & -39.31101127 & 0.00000000 & -23.38224068\end{array}$

maug-cc-pVTZ

$-116.05955769958263$

$\begin{array}{llll}\text { C } & 0.00649787 & 0.00000000 & 0.00328359\end{array}$

$\begin{array}{llll}\mathrm{H} & 0.00655115 & 0.00000000 & 1.07817004\end{array}$

$\mathrm{H} \quad 0.93733874 \quad 0.00000000 \quad-0.53424036$

$\mathrm{H} \quad-0.92444991 \quad-0.00000000 \quad-0.53412256$

$\begin{array}{llll}\mathrm{O} & -38.95883255 & -0.00000000 & -22.47853149\end{array}$

$\begin{array}{llll}\mathrm{H} & -39.74520191 & -0.00000000 & -21.92832748\end{array}$

$\begin{array}{llll}\mathrm{H} & -39.29874939 & 0.00000000 & -23.37545273\end{array}$

\section{tPBE}

maug-cc-pVDZ

$-116.10970783567717$

$\begin{array}{llll}\text { C } & 0.01679535 & 0.00000002 & 0.00929687\end{array}$

$\begin{array}{llll}\mathrm{H} & 0.01663305 & 0.00000013 & 1.10539912\end{array}$

$\mathrm{H} \quad 0.96613448 \quad-0.00000021 \quad-0.53860379$

$\begin{array}{llll}\mathrm{H} & -0.93237381 & 0.00000007 & -0.53889643\end{array}$

$\begin{array}{llll}\mathrm{O} & -38.96406250 & -0.00000003 & -22.48824903\end{array}$

$\begin{array}{llll}\mathrm{H} & -39.75575483 & 0.00000016 & -21.92595304\end{array}$

H $\quad-39.32421775 \quad-0.00000013 \quad-23.39221471$

maug-cc-pVTZ

$-116.14673911350870$

$\begin{array}{llll}\text { C } & 0.00882767 & 0.00000000 & 0.00496368\end{array}$

$\begin{array}{llll}\mathrm{H} & 0.00886254 & 0.00000032 & 1.08951156\end{array}$

$\mathrm{H} \quad 0.94805858 \quad-0.00000010 \quad-0.53733620$

$\mathrm{H} \quad-0.93043366 \quad-0.00000023 \quad-0.53728249$

$\begin{array}{llll}\mathrm{O} & -38.95767866 & -0.00000008 & -22.49456321\end{array}$

$\begin{array}{llll}\mathrm{H} & -39.72357910 & 0.00000009 & -21.90822851\end{array}$

$\begin{array}{llll}\mathrm{H} & -39.33090337 & -0.00000000 & -23.38628583\end{array}$ 


\section{Transition state}

\section{CASSCF}

maug-cc-pVDZ

$-115.57387091724694$

$\begin{array}{cccc}\mathrm{C} & 1.38539261 & 0.78487182 & -0.00005454 \\ \mathrm{O} & -0.56581348 & -0.87418501 & -0.00138860 \\ \mathrm{H} & 0.41474363 & -0.07765432 & -0.00080762 \\ \mathrm{H} & 1.94152598 & 0.56786385 & -0.90801867 \\ \mathrm{H} & 1.94023578 & 0.56811542 & 0.90875777 \\ \mathrm{H} & 0.93377771 & 1.77393452 & -0.00053826 \\ \mathrm{H} & -1.26065325 & -0.22203628 & 0.00204893\end{array}$

maug-cc-pVTZ

$-115.60743514707335$

$\begin{array}{cccc}\mathrm{C} & 1.38564595 & 0.78403361 & -0.00006148 \\ \mathrm{O} & -0.56565491 & -0.86881241 & -0.00147578 \\ \mathrm{H} & 0.41840781 & -0.07538461 & -0.00088054 \\ \mathrm{H} & 1.93784472 & 0.57028215 & -0.90038068 \\ \mathrm{H} & 1.93644680 & 0.57054048 & 0.90117268 \\ \mathrm{H} & 0.93637218 & 1.76408045 & -0.00057735 \\ \mathrm{H} & -1.25985356 & -0.22382966 & 0.00220215\end{array}$

\section{CASPT2}

maug-cc-pVDZ

$-115.91165069751712$

$\begin{array}{lrcc}\mathrm{C} & 1.36767194 & 0.77134476 & -0.00005560 \\ \mathrm{O} & -0.52632182 & -0.84618046 & -0.00136512 \\ \mathrm{H} & 0.44570513 & -0.11798152 & -0.00104221 \\ \mathrm{H} & 1.92533765 & 0.56047678 & -0.91825931 \\ \mathrm{H} & 1.92382563 & 0.56055863 & 0.91907761 \\ \mathrm{H} & 0.86981567 & 1.74630229 & -0.00054959 \\ \mathrm{H} & -1.21682521 & -0.15361047 & 0.00219322\end{array}$

maug-cc-pVTZ

$-116.02714555935653$

$\begin{array}{cccc}\mathrm{C} & 1.35766171 & 0.75277603 & -0.00007129 \\ \mathrm{O} & -0.58504321 & -0.87935677 & -0.00113475 \\ \mathrm{H} & 0.50530954 & -0.06873562 & -0.00075468 \\ \mathrm{H} & 1.93468408 & 0.57840993 & -0.89993466 \\ \mathrm{H} & 1.93349152 & 0.57844835 & 0.90056068 \\ \mathrm{H} & 0.88847106 & 1.72913644 & -0.00043332 \\ \mathrm{H} & -1.24536572 & -0.16976836 & 0.00176701\end{array}$




\section{tPBE}

maug-cc-pVDZ

$-116.08011676829986$

$\begin{array}{cccc}\mathrm{C} & 1.36669568 & 0.75025251 & 0.00087051 \\ \mathrm{O} & -0.64290739 & -0.91124282 & 0.01266375 \\ \mathrm{H} & 0.54121659 & -0.07462767 & 0.00950027 \\ \mathrm{H} & 1.95833748 & 0.59364354 & -0.91691707 \\ \mathrm{H} & 1.97427946 & 0.59354782 & 0.90821760 \\ \mathrm{H} & 0.88783842 & 1.74388523 & 0.00552562 \\ \mathrm{H} & -1.29625124 & -0.17454861 & -0.01986168\end{array}$

maug-cc-pVTZ

$-116.11411291397519$

$\begin{array}{cccc}\mathrm{C} & 1.36531342 & 0.75022333 & 0.00084583 \\ \mathrm{O} & -0.63257071 & -0.90293749 & 0.00821918 \\ \mathrm{H} & 0.53917149 & -0.06633934 & 0.00666958 \\ \mathrm{H} & 1.95006199 & 0.59365730 & -0.90842257 \\ \mathrm{H} & 1.96388877 & 0.58982056 & 0.90047386 \\ \mathrm{H} & 0.89196784 & 1.73450963 & 0.00688588 \\ \mathrm{H} & -1.28862380 & -0.17802400 & -0.01467277\end{array}$

\section{mod-CPO}

Reactants

CASSCF

maug-cc-pVDZ

$-115.66174871693273$

$\begin{array}{cccc}\mathrm{C} & 0.00351773 & 0.00000012 & -0.00816919 \\ \mathrm{H} & 0.00555269 & -0.00007530 & 1.10371194 \\ \mathrm{H} & 1.03270897 & 0.00003576 & -0.36953545 \\ \mathrm{H} & -0.51206021 & -0.89185688 & -0.36675891 \\ \mathrm{H} & -0.51207936 & 0.89189483 & -0.36663741 \\ \mathrm{O} & -23.57324230 & -0.00021262 & 9.40957196 \\ \mathrm{H} & -23.58484951 & 0.00021408 & 10.39248705\end{array}$

maug-cc-pVTZ

$-115.69643040369213$

$\begin{array}{cccc}\mathrm{C} & 0.00670469 & 0.00502579 & 0.00035514 \\ \mathrm{H} & 0.00423259 & 0.00143067 & 1.08249508 \\ \mathrm{H} & 1.02652094 & 0.00209573 & -0.36159992 \\ \mathrm{H} & -0.51249652 & -0.89496950 & -0.36759626 \\ \mathrm{H} & -0.50596448 & 0.88638546 & -0.36215885 \\ \mathrm{O} & -23.56828890 & -0.00062327 & 9.41366394 \\ \mathrm{H} & -23.59116031 & 0.00065512 & 10.38951087\end{array}$




\section{CASPT2}

maug-cc-pVDZ

$-115.92132174952444$

$\begin{array}{llll}\text { C } & 0.01166626 & 0.00042801 & -0.00611163\end{array}$

$\begin{array}{llll}\mathrm{H} & 0.02476927 & 0.01254757 & 1.09834452\end{array}$

H $\quad 1.04435097 \quad-0.01066477 \quad-0.38340129$

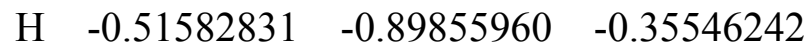

$\begin{array}{llll}\mathrm{H} & -0.50492586 & 0.89714992 & -0.37709235\end{array}$

$\begin{array}{llll}\mathrm{O} & -23.59913518 & 0.02856985 & 9.41983123\end{array}$

H $\quad-23.60134915 \quad-0.02947098 \quad 10.39856195$

maug-cc-pVTZ

$-116.03751855780253$

$\begin{array}{llll}\text { C } & 0.00128246 & 0.00081036 & -0.00003140\end{array}$

$\begin{array}{llll}\mathrm{H} & 0.00073636 & 0.00053132 & 1.08504223\end{array}$

$\mathrm{H} \quad 1.02447932 \quad 0.00135583 \quad-0.36122138$

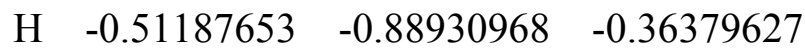

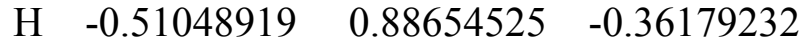

$\begin{array}{llll}\mathrm{O} & -23.57226707 & 0.00000251 & 9.41206195\end{array}$

H $\quad-23.57231736 \quad 0.00006442 \quad 10.38440718$

\section{tPBE}

maug-cc-pVDZ

$-116.08216159437416$

$\begin{array}{llll}\text { C } & 0.00312888 & 0.00000044 & 0.00005361\end{array}$

$\begin{array}{llll}\mathrm{H} & 0.00333492 & 0.00001183 & 1.10409146\end{array}$

H $\quad 1.04432566 \quad-0.00000111 \quad-0.37038499$

$\mathrm{H} \quad-0.51754690 \quad-0.90174788 \quad-0.37015486$

$\mathrm{H} \quad-0.51755241 \quad 0.90173631 \quad-0.37017691$

$\begin{array}{llll}\mathrm{O} & -23.57784586 & 0.00004369 & 9.40606050\end{array}$

$\mathrm{H} \quad-23.57829630 \quad-0.00004328 \quad 10.39518119$

maug-cc-pVTZ

$-116.11646167557998$

$\begin{array}{llll}\text { C } & 0.00124177 & -0.00109112 & -0.00121569\end{array}$

$\begin{array}{llll}\mathrm{H} & 0.00195166 & 0.00050605 & 1.09330799\end{array}$

$\mathrm{H} \quad 1.03358374 \quad 0.00083610 \quad-0.36488470$

$\begin{array}{llll}\mathrm{H} & -0.51402540 & -0.89393957 & -0.36595705\end{array}$

$\begin{array}{llll}\mathrm{H} & -0.51348727 & 0.89366637 & -0.36515902\end{array}$

$\begin{array}{llll}\mathrm{O} & -23.57511275 & -0.00014103 & 9.40840738\end{array}$

$\begin{array}{llll}\mathrm{H} & -23.57460375 & 0.00016320 & 10.39017110\end{array}$ 


\section{Products}

\section{CASSCF}

maug-cc-pVDZ

$-115.68828966874095$

C $\quad 0.01253365 \quad-0.00000341 \quad 0.00666124$

H $\quad 0.01149189 \quad-0.00000546 \quad 1.08647045$

$\mathrm{H} \quad 0.94819635 \quad 0.00000417 \quad-0.53234171$

$\mathrm{H} \quad-0.92208845 \quad 0.00000464 \quad-0.53414381$

$\begin{array}{llll}\mathrm{O} & -38.95734580 & 0.00000727 & -22.47704628\end{array}$

$\mathrm{H} \quad-39.76315364 \quad 0.00000840 \quad-21.93624457$

$\mathrm{H} \quad-39.30648001 \quad-0.00001560 \quad-23.38257632$

maug-cc-pVTZ

$-115.72521973003356$

C $\quad 0.00744250 \quad-0.00003686 \quad 0.00383192$

$\mathrm{H} \quad 0.00643674 \quad 0.00005112 \quad 1.07358556$

$\mathrm{H} \quad 0.93437904 \quad-0.00003495 \quad-0.53017428$

H $\quad-0.91848838 \quad 0.00001918 \quad-0.53191553$

$\begin{array}{lllll}\mathrm{O} & -38.95432251 & 0.00015496 & -22.47880537\end{array}$

$\mathrm{H} \quad-39.74657500 \quad-0.00007726 \quad-21.92875047$

$\begin{array}{llll}\mathrm{H} & -39.30571838 & -0.00007619 & -23.37699283\end{array}$

\section{CASPT2}

maug-cc-pVDZ

$-115.93795095917521$

$\begin{array}{llll}\mathrm{C} & 0.02105753 & 0.00000016 & 0.00910211\end{array}$

H $\quad 0.02233792 \quad-0.00012722 \quad 1.09908032$

$\mathrm{H} \quad 0.96434566 \quad 0.00012675 \quad-0.53702899$

$\mathrm{H} \quad-0.92357680 \quad 0.00000021 \quad-0.53477321$

$\begin{array}{llll}\text { O } & -39.01651409 & 0.00000649 & -22.38268116\end{array}$

$\mathrm{H} \quad-39.93430697 \quad-0.00002157 \quad-22.07762804$

$\mathrm{H} \quad-39.11018926 \quad 0.00001518 \quad-23.34529203$

maug-cc-pVTZ

$-116.05744062326660$

$\begin{array}{llll}\text { C } & 0.01191889 & -0.00000042 & 0.00395396\end{array}$

$\begin{array}{llll}\mathrm{H} & 0.01541717 & 0.00001553 & 1.07884844\end{array}$

$\mathrm{H} \quad 0.94104942 \quad-0.00001360 \quad-0.53654917$

$\mathrm{H} \quad-0.92073678 \quad-0.00000114 \quad-0.53050825$

$\begin{array}{llll}\mathrm{O} & -39.01006908 & -0.00001403 & -22.38089578\end{array}$

H $\quad-39.91755742 \quad-0.00001318 \quad-22.06664894$

$\mathrm{H} \quad-39.09686820 \quad 0.00002686 \quad-23.33742127$ 


\section{tPBE}

maug-cc-pVDZ

$-116.09524059310134$

$\begin{array}{llll}\text { C } & 0.01561460 & 0.00000020 & 0.00884969\end{array}$

$\mathrm{H} \quad 0.01566089 \quad 0.00000055 \quad 1.10495304$

$\mathrm{H} \quad 0.96484742 \quad 0.00000044 \quad-0.53923777$

$\mathrm{H} \quad-0.93366100 \quad-0.00000120 \quad-0.53916392$

$\begin{array}{llll}\mathrm{O} & -38.95974695 & -0.00000145 & -22.49811680\end{array}$

$\mathrm{H} \quad-39.73807664-0.00000017 \quad-21.91197202$

$\mathrm{H} \quad-39.34148431 \quad 0.00000163 \quad-23.39453321$

maug-cc-pVTZ

$-116.13165913848209$

$\begin{array}{llll}\text { C } & 0.00840352 & 0.00000015 & 0.00475864\end{array}$

$\begin{array}{llll}\mathrm{H} & 0.00841253 & 0.00000173 & 1.08930703\end{array}$

$\mathrm{H} \quad 0.94764784 \quad-0.00000402 \quad-0.53751955$

$\mathrm{H} \quad-0.93084572 \quad 0.00000211 \quad-0.53750941$

$\begin{array}{lllll}\text { O } & -38.95669499 & 0.00001043 & -22.49673347\end{array}$

H $-39.72124825 \quad-0.00000693 \quad-21.90201783$

H $\quad-39.33252094 \quad-0.00000347 \quad-23.38950642$

\section{Transition state}

\section{CASSCF}

maug-cc-pVDZ

$-115.63889426996563$

$\begin{array}{cccc}\mathrm{C} & 1.37445822 & 0.77214013 & -0.00006655 \\ \mathrm{O} & -0.57799995 & -0.89885631 & -0.00144651 \\ \mathrm{H} & 0.45396012 & -0.05654921 & -0.00080315 \\ \mathrm{H} & 1.94130424 & 0.57081195 & -0.90556617 \\ \mathrm{H} & 1.93997736 & 0.57102354 & 0.90630770 \\ \mathrm{H} & 0.92321272 & 1.76185362 & -0.00054216 \\ \mathrm{H} & -1.26570373 & -0.19951372 & 0.00211585\end{array}$

maug-cc-pVTZ

$-115.67338717733064$

$\begin{array}{cccc}\mathrm{C} & 1.37321132 & 0.77052878 & -0.00006873 \\ \mathrm{O} & -0.57769120 & -0.89399704 & -0.00142786 \\ \mathrm{H} & 0.45827023 & -0.05551127 & -0.00080525 \\ \mathrm{H} & 1.93631865 & 0.57301887 & -0.89807014 \\ \mathrm{H} & 1.93499240 & 0.57321557 & 0.89880355 \\ \mathrm{H} & 0.92342026 & 1.75098443 & -0.00053554 \\ \mathrm{H} & -1.25931267 & -0.19732934 & 0.00210297\end{array}$




\section{CASPT2}

maug-cc-pVDZ

$-115.90832236456852$

$\begin{array}{cccc}\mathrm{C} & 1.36112393 & 0.76192773 & -0.00007166 \\ \mathrm{O} & -0.55820100 & -0.87543486 & -0.00143570 \\ \mathrm{H} & 0.46435017 & -0.07833710 & -0.00081955 \\ \mathrm{H} & 1.93477004 & 0.56565464 & -0.91498340 \\ \mathrm{H} & 1.93338273 & 0.56581460 & 0.91573694 \\ \mathrm{H} & 0.89123332 & 1.75437103 & -0.00054101 \\ \mathrm{H} & -1.23745021 & -0.17308603 & 0.00211339\end{array}$

maug-cc-pVTZ

$-116.02736363576464$

$\begin{array}{llll}\text { C } & 1.36186479 & 0.76080239 & -0.00006632\end{array}$

$\begin{array}{llll}\mathrm{O} & -0.55837815 & -0.86672705 & -0.00139007\end{array}$

$\mathrm{H} \quad 0.47624400 \quad-0.07313792 \quad-0.00087766$

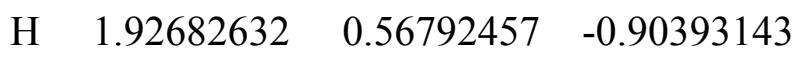

$\begin{array}{llll}\mathrm{H} & 1.92543297 & 0.56805527 & 0.90469240\end{array}$

$\mathrm{H} \quad 0.89112932 \quad 1.73669853 \quad-0.00054396$

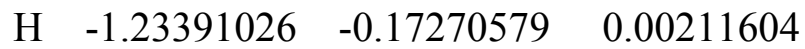

\section{tPBE}

maug-cc-pVDZ

$-116.07516128002950$

$\begin{array}{llll}\text { C } & 1.37463965 & 0.75842011 & -0.00226472\end{array}$

$\begin{array}{llll}\mathrm{O} & -0.65673515 & -0.92175945 & 0.01278090\end{array}$

$\begin{array}{llll}\mathrm{H} & 0.53303219 & -0.04531977 & -0.06120691\end{array}$

$\begin{array}{llll}\mathrm{H} & 1.99586300 & 0.59998791 & -0.90057811\end{array}$

$\begin{array}{llll}\mathrm{H} & 1.94496411 & 0.57602877 & 0.92398364\end{array}$

$\begin{array}{llll}\mathrm{H} & 0.90689914 & 1.75726641 & -0.00766399\end{array}$

$\begin{array}{llll}\mathrm{H} & -1.16205495 & -0.38297197 & 0.66780718\end{array}$ maug-cc-pVTZ

$-116.10908016460898$

$\begin{array}{llll}\text { C } & 1.37526246 & 0.75518386 & 0.00107563\end{array}$

$\begin{array}{llll}\mathrm{O} & -0.65163863 & -0.91224731 & 0.01348771\end{array}$

$\mathrm{H} \quad 0.54945206 \quad-0.05733726 \quad 0.00933527$

$\begin{array}{llll}\mathrm{H} & 1.95153339 & 0.59999847 & -0.91397752\end{array}$

$\begin{array}{llll}\mathrm{H} & 1.97817412 & 0.58731303 & 0.89660690\end{array}$

$\begin{array}{llll}\mathrm{H} & 0.90082315 & 1.73898712 & 0.01541568\end{array}$

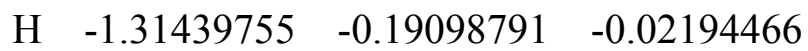



R2: $\mathrm{H}+\mathrm{OH} \rightarrow \mathrm{O}+\mathrm{H}_{2}$
nom-CPO
Reactants
CASSCF
maug-cc-pVDZ
$-75.919915019533121$
$\begin{array}{rrrc}\mathrm{O} & 0.00030545 & -0.00057828 & 0.18177344 \\ \mathrm{H} & -0.00068563 & 0.00129706 & -0.79906412 \\ \mathrm{H} & 0.03909018 & -0.07394278 & -26.68574032\end{array}$
maug-cc-pVTZ
$-75.939478134404126$
H $\quad-24.01521707 \quad 0.00000000 \quad-0.87580548$
O $\quad-0.48877480 \quad 0.00000000 \quad-0.22672862$
$\mathrm{H} \quad-0.49600814 \quad 0.00000000 \quad-1.20063390$

\section{CASPT2}

maug-cc-pVDZ

$-76.057481935961505$

$\begin{array}{llll}\text { O } & 0.00030318 & -0.00057399 & 0.18127192\end{array}$

$\mathrm{H} \quad-0.00068313 \quad 0.00129234 \quad-0.79866566$

H $\quad 0.03908995 \quad-0.07394235 \quad-26.68563726$

maug-cc-pVTZ

$-76.125674687187384$

$\mathrm{H} \quad-25.11987781 \quad 0.00000000 \quad-0.88338873$

O $\quad 0.06241453 \quad 0.00000000 \quad-0.22414781$

$\mathrm{H} \quad 0.05746328 \quad 0.00000000 \quad-1.19563147$

\section{tPBE}

maug-cc-pVDZ

$-76.103903581147236$

$\begin{array}{llll}\mathrm{O} & 0.05754457 & -0.00070458 & 0.28442163\end{array}$

$\mathrm{H} \quad-25.11264579 \quad 0.00165474 \quad-0.88541270$

H $\quad 0.05503722 \quad-0.00082616 \quad-0.70217694$

maug-cc-pVTZ

$-75.956705969054113$

H $\quad-25.20164926 \quad 0.00166417 \quad-0.88265818$

$\begin{array}{llll}\text { O } & 0.09063507 & -0.00058021 & 1.31714600\end{array}$

H $\quad 0.11101419 \quad-0.00108396 \quad-2.73765582$ 


\section{Products}

\section{CASSCF}

maug-cc-pVDZ

$-75.938381905325457$

$\begin{array}{llll}\mathrm{H} & -0.00078027 & 0.00147596 & -0.92114931\end{array}$

$\begin{array}{llll}\mathrm{O} & 0.00036455 & -0.00068958 & 23.30953691\end{array}$

$\mathrm{H} \quad 0.00041572 \quad-0.00078637 \quad-1.69155560$

maug-cc-pVTZ

$-75.957910771816742$

$\begin{array}{llll}\mathrm{H} & -0.00076858 & 0.00145385 & -0.92619153\end{array}$

$\begin{array}{llll}\mathrm{O} & 0.00036444 & -0.00068938 & 23.30449455\end{array}$

$\mathrm{H} \quad 0.00040414 \quad-0.00076447 \quad-1.68147101$

\section{CASPT2}

maug-cc-pVDZ

$-76.063719321974503$

$\begin{array}{llll}\mathrm{H} & -0.00077552 & 0.00146697 & -0.92347563\end{array}$

$\begin{array}{llll}\mathrm{O} & 0.00036452 & -0.00068953 & 23.30721255\end{array}$

$\mathrm{H} \quad 0.00041099 \quad-0.00077744 \quad-1.68690492$

maug-cc-pVTZ

$-76.129484169776703$

$\begin{array}{llll}\mathrm{H} & -0.00076091 & 0.00143933 & -0.92967703\end{array}$

$\begin{array}{llll}\mathrm{O} & 0.00036438 & -0.00068926 & 23.30101038\end{array}$

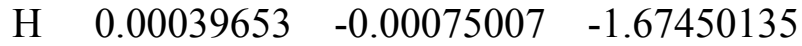

\section{tPBE}

maug-cc-pVDZ

$-76.143143529663874$

$\begin{array}{llll}\mathrm{H} & -0.00085710 & 0.00162130 & -0.92101241\end{array}$

$\begin{array}{llll}\mathrm{O} & 0.00036708 & -0.00069437 & 23.30967393\end{array}$

$\mathrm{H} \quad 0.00049002 \quad-0.00092692 \quad-1.69182952$

maug-cc-pVTZ

$-76.162200560839537$

$\begin{array}{llll}\mathrm{H} & -0.00077668 & 0.00146916 & -0.92707621\end{array}$

$\begin{array}{llll}\mathrm{O} & 0.00036473 & -0.00068993 & 23.30360979\end{array}$

$\mathrm{H} \quad 0.00041194 \quad-0.00077923 \quad-1.67970158$ 


\section{Transition state}

\section{CASSCF}

maug-cc-pVDZ

$-75.839341011144015$

$\mathrm{H} \quad-0.00041694 \quad 0.00078868 \quad-0.97185653$

$\begin{array}{llll}\mathrm{O} & 0.00024037 & -0.00045468 & 0.48240279\end{array}$

$\mathrm{H} \quad 0.00017657 \quad-0.00033400 \quad-1.81371427$

maug-cc-pVTZ

$-75.859210699813033$

$\begin{array}{lllr}\mathrm{H} & 0.00000000 & 0.00000000 & -0.96767217 \\ \mathrm{O} & 0.00000000 & 0.00000000 & 0.46634590 \\ \mathrm{H} & 0.00000000 & 0.00000000 & -1.80184173\end{array}$

\section{CASPT2}

maug-cc-pVDZ

$-76.038591809195580$

$\begin{array}{llll}\mathrm{H} & 0.00001174 & -0.00002221 & -0.87007148\end{array}$

$\begin{array}{llll}\mathrm{O} & -0.00000726 & 0.00001373 & 0.34877186\end{array}$

$\mathrm{H} \quad-0.00000448 \quad 0.00000847 \quad-1.78186838$

maug-cc-pVTZ

$-76.106444642971098$

$\begin{array}{llll}\mathrm{H} & -0.00001726 & 0.00003265 & -0.87760216\end{array}$

$\begin{array}{llll}\mathrm{O} & 0.00000656 & -0.00001241 & 0.34231708\end{array}$

H $\quad 0.00001070 \quad-0.00002023 \quad-1.76788292$

\section{tPBE}

maug-cc-pVDZ

$-76.129018467812841$

$\begin{array}{llll}\mathrm{H} & -0.00020790 & 0.00039327 & -0.93832846\end{array}$

$\begin{array}{llll}\text { O } & 0.00002739 & -0.00005181 & 0.41741752\end{array}$

$\mathrm{H} \quad 0.00018051 \quad-0.00034146 \quad-1.78225706$

maug-cc-pVTZ

$-76.146917668194291$

H $\quad 0.00000022 \quad-0.00000042 \quad-0.93475261$

$\begin{array}{llll}\mathrm{O} & 0.00001056 & -0.00001998 & 0.40024717\end{array}$

$\mathrm{H} \quad-0.00001079 \quad 0.00002041 \quad-1.76866256$ 


\section{mod-CPO}

Reactants

CASSCF

maug-cc-pVDZ

$-75.946376713651645$

$\begin{array}{rrrc}\mathrm{O} & 0.00030851 & -0.00058406 & 0.18320449 \\ \mathrm{H} & -0.00068985 & 0.00130504 & -0.79977965 \\ \mathrm{H} & 0.03909134 & -0.07394498 & -26.68645584\end{array}$

maug-cc-pVTZ

$-75.967039763982342$

$\begin{array}{llll}\mathrm{O} & 0.00030391 & -0.00057537 & 0.17863901\end{array}$

$\mathrm{H} \quad-0.00068175 \quad 0.00128972 \quad-0.79749606$

H $\quad 0.03908784 \quad-0.07393835 \quad-26.68417394$

\section{CASPT2}

maug-cc-pVDZ

$-76.057998086288109$

$\begin{array}{llll}\text { O } & 0.00030674 & -0.00058073 & 0.18160928\end{array}$

$\mathrm{H} \quad-0.00068706 \quad 0.00129977 \quad-0.79884722$

H $\quad 0.03909032 \quad-0.07394304 \quad-26.68579306$

maug-cc-pVTZ

$-76.124960384353130$

$\begin{array}{llll}\text { O } & 0.00039080 & -0.00073974 & 0.17619010\end{array}$

$\mathrm{H} \quad-0.00077028 \quad 0.00145721 \quad-0.79627807$

H $\quad 0.03908949 \quad-0.07394147 \quad-26.68294303$

tPBE

maug-cc-pVDZ

$-76.140260574277463$

$\begin{array}{llll}\text { O } & 0.00017551 & -0.00033248 & 0.26069640\end{array}$

$\mathrm{H} \quad-0.00077742 \quad 0.00147069 \quad-0.72843498$

$\mathrm{H} \quad 0.03931191 \quad-0.07436221 \quad-26.83529242$

maug-cc-pVTZ

$-76.159373119450237$

$\begin{array}{rrrc}\mathrm{O} & 0.00033298 & -0.00063035 & 0.18239138 \\ \mathrm{H} & -0.00071473 & 0.00135210 & -0.79937183 \\ \mathrm{H} & 0.03909175 & -0.07394575 & -26.68605056\end{array}$

\section{Products}

CASSCF

maug-cc-pVDZ

$-75.951049029141728$

H $\quad-0.00078025 \quad 0.00147593 \quad-0.92115045$

O $\quad 0.00036455 \quad-0.00068958 \quad 23.30953747$

$\mathrm{H} \quad 0.00041570 \quad-0.00078635 \quad-1.69155503$ 


\begin{tabular}{lrrl}
\multicolumn{4}{c}{ maug-cc-pVTZ } \\
-75.971455237960598 & \\
H & -0.00076858 & 0.00145385 & -0.92619281 \\
O & 0.00036444 & -0.00068938 & 23.30449498 \\
H & 0.00040414 & -0.00076447 & -1.68147018
\end{tabular}

\section{CASPT2}

maug-cc-pVDZ

$$
-76.064544183832737
$$

$\begin{array}{rrrr}\mathrm{H} & -0.00077550 & 0.00146694 & -0.92347580 \\ \mathrm{O} & 0.00036452 & -0.00068953 & 23.30721277 \\ \mathrm{H} & 0.00041098 & -0.00077741 & -1.68690497\end{array}$

maug-cc-pVTZ

$-76.129605906692817$
$\mathrm{H} \quad-0.00076087 \quad 0.00143925$
$-0.92967809$
O $\quad 0.00036438 \quad-0.00068926 \quad 23.30101038$
H $\quad 0.00039649 \quad-0.00074999 \quad-1.67450029$

\section{tPBE}

maug-cc-pVDZ

$-76.143458097328846$

$\begin{array}{rrrr}\mathrm{H} & -0.00085689 & 0.00162089 & -0.92101305 \\ \mathrm{O} & 0.00036708 & -0.00069436 & 23.30967490 \\ \mathrm{H} & 0.00048981 & -0.00092653 & -1.69182986\end{array}$

maug-cc-pVTZ

$-76.162790299100067$
H $\quad-0.00077663$
$0.00146906-0.92707589$
O $\quad 0.00036473 \quad-0.00068992 \quad 23.30361192$
H $\quad 0.00041189 \quad-0.00077914 \quad-1.67970404$

\section{Transition state}

\section{CASSCF}

maug-cc-pVDZ

$-75.856286131896709$

$\begin{array}{lllr}\mathrm{H} & 0.00000000 & 0.00000000 & -1.01205628 \\ \mathrm{O} & 0.00000000 & 0.00000000 & 0.53663316 \\ \mathrm{H} & 0.00000000 & 0.00000000 & -1.82774488\end{array}$

maug-cc-pVTZ

$-75.935477252846923$
$\mathrm{H} \quad-0.01512522$
0.02861089
$-0.86930807$
$\begin{array}{llll}\mathrm{O} & 0.00620514 & -0.01173764 & 0.35671378\end{array}$
H $\quad 0.00892009 \quad-0.01687325 \quad-1.79057371$

\section{CASPT2}

maug-cc-pVDZ

$-76.039634513940641$
H 0.00929398
$-0.01758050$
$-0.86112734$
O $\quad-0.00369427$
0.00698809
0.34404520
$\mathrm{H} \quad-0.00559971$
0.01059242
$-1.78608586$ 
maug-cc-pVTZ

$-76.106999841518345$
H $\quad-0.00892413$
0.01688088
$-0.86685398$
$\begin{array}{llll}\text { O } & 0.00368807 & -0.00697635 & 0.33640654\end{array}$
H $\quad 0.00523606 \quad-0.00990453 \quad-1.77272056$

tPBE

maug-cc-pVDZ

$-76.128429272269202$

$\begin{array}{cccc}\mathrm{H} & 0.00698702 & -0.01321666 & -0.85865564 \\ \mathrm{O} & -0.00284695 & 0.00538530 & 0.34697658 \\ \mathrm{H} & -0.00414007 & 0.00783136 & -1.79148894 \\ \text { maug-cc-pVTZ } & & \\ -76.146378228731123 & \\ \mathrm{H} & -0.00852930 & 0.01613402 & -0.85031497 \\ \mathrm{O} & 0.00346836 & -0.00656074 & 0.33261749 \\ \mathrm{H} & 0.00506094 & -0.00957328 & -1.78547052\end{array}$

R3: $\mathrm{H}+\mathrm{H}_{2} \mathrm{~S} \rightarrow \mathrm{H}_{2}+\mathrm{HS}$

nom-CPO

Reactants

CASSCF

maug-cc-pVDZ

$-399.21198448171293$

$\begin{array}{crcc}\mathrm{S} & -0.03240108 & 0.17568336 & 0.00000000 \\ \mathrm{H} & 1.26357557 & -0.16180891 & 0.00000000 \\ \mathrm{H} & -0.45854892 & -1.12020741 & 0.00000000 \\ \mathrm{H} & -7.65841356 & -30.24012605 & 0.00000000 \\ \text { maug-cc-pVTZ } & & \end{array}$

maug-cc-pVTZ

$-399.22891523209353$
S -0.03220516
0.15936141
0.00000000
$\mathrm{H} \quad 1.26970077$
$-0.21211660$
0.00000000
$\mathrm{H} \quad-0.47814630 \quad-1.09342941$
0.00000000
H $\quad-7.64513731 \quad-30.20027441$
0.00000000

\section{CASPT2}

maug-cc-pVDZ

$-399.34423535217007$
S -0.08603175
0.16206617
0.00000000
$\mathrm{H} \quad 1.24291723 \quad-0.05686488 \quad 0.00000000$
$\mathrm{H} \quad-0.36335115 \quad-1.16389296 \quad 0.00000000$
$\mathrm{H} \quad-7.67932233 \quad-30.28776734 \quad 0.00000000$
maug-cc-pVTZ
$-399.40568961192452$
$\begin{array}{lccc}\mathrm{S} & 0.05183702 & 0.18163136 & 0.00000000 \\ \mathrm{H} & 1.25707661 & -0.40880209 & 0.00000000 \\ \mathrm{H} & -0.58246193 & -0.99329723 & 0.00000000 \\ \mathrm{H} & -7.61223969 & -30.12599104 & 0.00000000\end{array}$ 


\section{tPBE}

maug-cc-pVDZ $-399.70685953684523$
S $\quad-0.06197647$
0.17646028
0.00000000
$\mathrm{H} \quad 1.26355745 \quad-0.13948205$
0.00000000
$\mathrm{H} \quad-0.42465325 \quad-1.13665930 \quad 0.00000000$
$\mathrm{H} \quad-7.66271572 \quad-30.24677794 \quad 0.00000000$
maug-cc-pVTZ
$-399.72261838336749$

$\begin{array}{lrcc}\mathrm{S} & -0.06450179 & 0.16847680 & 0.00000000 \\ \mathrm{H} & 1.25260868 & -0.13136628 & 0.00000000 \\ \mathrm{H} & -0.41067603 & -1.13797191 & 0.00000000 \\ \mathrm{H} & -7.66321886 & -30.24559761 & 0.00000000\end{array}$

\section{Products}

CASSCF

maug-cc-pVDZ

$-399.23428122689853$
$\mathrm{S} \quad-0.04222340 \quad-0.41391154$
0.00000000
H $\quad 1.23789646 \quad-0.81920003 \quad 0.00000000$
H $-13.63067722 \quad-54.72821745 \quad 0.00000000$
H $\quad-13.77899584 \quad-55.48422098 \quad 0.00000000$
maug-cc-pVTZ
$-399.25153831420630$

$\begin{array}{lcll}\mathrm{S} & -0.03724998 & -0.41448459 & 0.00000000 \\ \mathrm{H} & 1.23201489 & -0.82183150 & 0.00000000 \\ \mathrm{H} & -13.63156189 & -54.73405598 & 0.00000000 \\ \mathrm{H} & -13.77720302 & -55.47517792 & 0.00000000\end{array}$

\section{CASPT2}

maug-cc-pVDZ

$-399.36485070875875$

\begin{tabular}{|c|c|c|c|}
\hline $\mathrm{S}$ & -0.04700341 & -0.41602257 & 0.00000000 \\
\hline $\mathrm{H}$ & 1.23920645 & -0.83002457 & 0.00000000 \\
\hline $\mathrm{H}$ & -13.62993552 & -54.72510807 & 0.00000000 \\
\hline $\mathrm{H}$ & -13.77626753 & -55.47439480 & 0.00000000 \\
\hline \multicolumn{4}{|c|}{ maug-cc-pVTZ } \\
\hline \multicolumn{4}{|c|}{-399.42703363747131} \\
\hline S & -0.03975130 & -0.41261228 & 0.00000000 \\
\hline $\mathrm{H}$ & 1.23455338 & -0.82347300 & 0.00000000 \\
\hline $\mathrm{H}$ & -13.63248933 & -54.73932464 & 0.00000000 \\
\hline 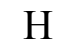 & -13.77631275 & -55.47014008 & 0.00000000 \\
\hline
\end{tabular}




\section{tPBE}

maug-cc-pVDZ

$-399.72410917068186$

S $\quad-0.05307994 \quad-0.40737670 \quad 0.00000000$

$\mathrm{H} \quad 1.24687511 \quad-0.83265479 \quad 0.00000000$

$\mathrm{H} \quad-13.63661513 \quad-54.72327744 \quad 0.00000000$

$\mathrm{H} \quad-13.77118004 \quad-55.48224107 \quad 0.00000000$

maug-cc-pVTZ

$-399.74106653202563$

$\begin{array}{lcll}\mathrm{S} & -0.05097087 & -0.42100145 & 0.00000000 \\ \mathrm{H} & 1.24476280 & -0.82014724 & 0.00000000 \\ \mathrm{H} & -13.63834724 & -54.73166021 & 0.00000000 \\ \mathrm{H} & -13.76944469 & -55.47274110 & 0.00000000\end{array}$

Transition state

CASSCF

maug-cc-pVDZ

$-399.19327309470629$

$\begin{array}{llll}\mathrm{H} & 1.28161309 & -0.17611622 & 0.00000000 \\ \mathrm{~S} & 0.00841554 & 0.24357009 & 0.00000000 \\ \mathrm{H} & -0.50138665 & -1.17576557 & 0.00000000 \\ \mathrm{H} & -0.78864199 & -2.23398231 & 0.00000000\end{array}$

maug-cc-pVTZ

$-399.20944366355394$

H $\quad 1.27139004 \quad-0.18401108 \quad 0.00000000$

S $\quad 0.00940466 \quad 0.23893957 \quad 0.00000000$

$\mathrm{H} \quad-0.50194537 \quad-1.17284815 \quad 0.00000000$

$\mathrm{H} \quad-0.77884932 \quad-2.22437434 \quad 0.00000000$

\section{CASPT2}

maug-cc-pVDZ $-399.33473109591353$

$\begin{array}{cccc}\mathrm{H} & 1.27377924 & -0.19463515 & 0.00000000 \\ \mathrm{~S} & -0.00509152 & 0.23348700 & 0.00000000 \\ \mathrm{H} & -0.48669429 & -1.12863535 & 0.00000000 \\ \mathrm{H} & -0.78199343 & -2.25251051 & 0.00000000 \\ \text { maug-cc-pVTZ } & & \\ -399.39750876772354 & \\ \mathrm{H} & 1.25900599 & -0.20844609 & 0.00000000 \\ \mathrm{~S} & -0.00408856 & 0.22954595 & 0.00000000 \\ \mathrm{H} & -0.48640427 & -1.11494395 & 0.00000000 \\ \mathrm{H} & -0.76851315 & -2.24844993 & 0.00000000\end{array}$




\section{tPBE}

maug-cc-pVDZ $-399.70077406997132$

$\begin{array}{cccc}\mathrm{H} & 1.29439376 & -0.17130426 & 0.00000000 \\ \mathrm{~S} & -0.00270833 & 0.24938368 & 0.00000000 \\ \mathrm{H} & -0.46704038 & -1.09285923 & 0.00000000 \\ \mathrm{H} & -0.82464505 & -2.32751419 & 0.00000000 \\ \text { maug-cc-pVTZ } & & \\ -399.71564447953051 & \\ \mathrm{H} & 1.28049006 & -0.18496622 & 0.00000000 \\ \mathrm{~S} & -0.00237282 & 0.24295808 & 0.00000000 \\ \mathrm{H} & -0.47103558 & -1.08971563 & 0.00000000 \\ \mathrm{H} & -0.80708167 & -2.31057025 & 0.00000000\end{array}$

\section{mod-CPO}

Reactants

CASSCF

maug-cc-pVDZ

$-399.24282272082212$

$\begin{array}{cccc}\mathrm{S} & -0.04296951 & 0.18531555 & 0.00000000 \\ \mathrm{H} & 1.25328645 & -0.19138469 & 0.00000000 \\ \mathrm{H} & -0.43972785 & -1.10515605 & 0.00000000 \\ \mathrm{H} & -7.65637708 & -30.23523382 & 0.00000000 \\ \text { maug-cc-pVTZ } & & \end{array}$

$-399.26327806207041$
S $\quad-0.04214727$
0.17427996
0.00000000
$\mathrm{H} \quad 1.24521266$
$-0.19258851$
0.00000000
$\mathrm{H} \quad-0.43555865 \quad-1.10521850 \quad 0.00000000$
H $\quad-7.65329474 \quad-30.22293196$
0.00000000

\section{CASPT2}

maug-cc-pVDZ

$-399.34808753076192$
S $\quad-0.10238685$
0.15253524
0.00000000
H $\quad 1.23419899 \quad-0.05961599 \quad 0.00000000$
$\mathrm{H} \quad-0.34943005 \quad-1.17808525 \quad 0.00000000$
H $\quad-12.72100009 \quad-50.81090700 \quad 0.00000000$
maug-cc-pVTZ
$-399.41046840913515$

$\begin{array}{cccc}\mathrm{S} & -0.03743907 & 0.17383606 & 0.00000000 \\ \mathrm{H} & 1.24842272 & -0.19950346 & 0.00000000 \\ \mathrm{H} & -0.44508694 & -1.10155100 & 0.00000000 \\ \mathrm{H} & -7.65168472 & -30.21924061 & 0.00000000\end{array}$




\section{tPBE}

maug-cc-pVDZ

$-399.69881086167658$

$\begin{array}{cccc}\mathrm{S} & -0.02616010 & 0.18340147 & 0.00000000 \\ \mathrm{H} & 1.29134109 & -0.17517984 & 0.00000000 \\ \mathrm{H} & -0.49436469 & -1.11404235 & 0.00000000 \\ \mathrm{H} & -7.65660430 & -30.24063830 & 0.00000000 \\ \text { maug-cc-pVTZ } & & \\ -399.71234115101868 & \\ \mathrm{~S} & -0.04468033 & 0.17664016 & 0.00000000 \\ \mathrm{H} & 1.26455394 & -0.17136107 & 0.00000000 \\ \mathrm{H} & -0.44853479 & -1.11667060 & 0.00000000 \\ \mathrm{H} & -7.65712681 & -30.23506749 & 0.00000000\end{array}$

Products

CASSCF

maug-cc-pVDZ $-399.22385281325177$

$\begin{array}{llll}\mathrm{H} & 1.26685467 & -0.21293999 & 0.00000000 \\ \mathrm{~S} & 0.00054916 & 0.25426811 & 0.00000000 \\ \mathrm{H} & -0.51368186 & -1.16095702 & 0.00000000 \\ \mathrm{H} & -0.75372197 & -2.22266512 & 0.00000000\end{array}$

maug-cc-pVTZ

$-399.28260143637624$

S $\quad-0.04141661 \quad-0.41185073 \quad 0.00000000$

H $\quad 1.23653046 \quad-0.82308590 \quad 0.00000000$

$\mathrm{H} \quad-13.63170501 \quad-54.73476546 \quad 0.00000000$

$\mathrm{H} \quad-13.77740884 \quad-55.47584791 \quad 0.00000000$

\section{CASPT2}

maug-cc-pVDZ $-399.36867250257512$
$\mathrm{S} \quad-0.01534758 \quad-0.33340068$
0.00000000
$\mathrm{H} \quad 1.19654406 \quad-0.94522758 \quad 0.00000000$
H $-13.63923862 \quad-54.70481797 \quad 0.00000000$
$\mathrm{H} \quad-13.75595785 \quad-55.46210378 \quad 0.00000000$
maug-cc-pVTZ
$-399.43069551032977$

$\begin{array}{lcll}\mathrm{S} & -0.04337978 & -0.41377706 & 0.00000000 \\ \mathrm{H} & 1.23715073 & -0.82625947 & 0.00000000 \\ \mathrm{H} & -13.63208246 & -54.73732538 & 0.00000000 \\ \mathrm{H} & -13.77568848 & -55.46818809 & 0.00000000\end{array}$




\section{tPBE}

maug-cc-pVDZ

$-399.71864012324033$

S $\quad-0.06085748 \quad-0.40043515 \quad 0.00000000$

$\mathrm{H} \quad 1.25784724 \quad-0.82725384 \quad 0.00000000$

H $-13.63134326 \quad-54.73069851 \quad 0.00000000$

$\mathrm{H} \quad-13.77964650 \quad-55.48716250 \quad 0.00000000$

maug-cc-pVTZ

$-399.73395387206585$

$\begin{array}{lcll}\mathrm{S} & -0.04471855 & -0.37418312 & 0.00000000 \\ \mathrm{H} & 1.24315738 & -0.84481561 & 0.00000000 \\ \mathrm{H} & -13.62067757 & -54.74680837 & 0.00000000 \\ \mathrm{H} & -13.79176127 & -55.47974290 & 0.00000000\end{array}$

Transition state

CASSCF

maug-cc-pVDZ

$-399.22385281325177$

$\begin{array}{llll}\mathrm{H} & 1.26685467 & -0.21293999 & 0.00000000 \\ \mathrm{~S} & 0.00054916 & 0.25426811 & 0.00000000 \\ \mathrm{H} & -0.51368186 & -1.16095702 & 0.00000000 \\ \mathrm{H} & -0.75372197 & -2.22266512 & 0.00000000\end{array}$

maug-cc-pVTZ

$-399.24279460454852$

$\begin{array}{llll}\mathrm{H} & 1.25276646 & -0.22593178 & 0.00000000 \\ \mathrm{~S} & 0.00140763 & 0.24901466 & 0.00000000 \\ \mathrm{H} & -0.51651186 & -1.15774658 & 0.00000000 \\ \mathrm{H} & -0.73766224 & -2.20763031 & 0.00000000\end{array}$

CASPT2

maug-cc-pVDZ

$-399.33888847711671$

$\begin{array}{llll}\mathrm{H} & 1.27331931 & -0.20309004 & 0.00000000 \\ \mathrm{~S} & -0.00757406 & 0.23783234 & 0.00000000 \\ \mathrm{H} & -0.49126480 & -1.12222264 & 0.00000000 \\ \mathrm{H} & -0.77448045 & -2.25481367 & 0.00000000\end{array}$

maug-cc-pVTZ

$-399.40208498066983$

$\begin{array}{lccc}\mathrm{H} & 1.25408147 & -0.22111368 & 0.00000000 \\ \mathrm{~S} & -0.00677898 & 0.23264012 & 0.00000000 \\ \mathrm{H} & -0.49153414 & -1.11058194 & 0.00000000 \\ \mathrm{H} & -0.75576835 & -2.24323851 & 0.00000000\end{array}$




\section{tPBE}

maug-cc-pVDZ $-399.69380530267358$

$\begin{array}{llll}\text { H } & 1.31873688 & -0.14102997 & 0.00000000 \\ \text { S } & 0.00488622 & 0.25062525 & 0.00000000 \\ \text { H } & -0.47161534 & -1.08769372 & 0.00000000 \\ \text { H } & -0.85200776 & -2.36419557 & 0.00000000\end{array}$
maug-cc-pVTZ

$-399.70685788889091$

$\begin{array}{llll}\mathrm{H} & 1.31713842 & -0.13053943 & 0.00000000 \\ \mathrm{~S} & 0.02116340 & 0.25293107 & 0.00000000 \\ \mathrm{H} & -0.47552681 & -1.07308597 & 0.00000000 \\ \mathrm{H} & -0.86277500 & -2.39159969 & 0.00000000\end{array}$

R4: $\mathrm{H}+\mathrm{N}_{2} \mathrm{O} \rightarrow \mathrm{N}_{2}+\mathrm{OH}$

nom-CPO, mod-CPO

Reactants

CASSCF

maug-cc-pVDZ

$-184.40694340898921$

$\begin{array}{llll}\mathrm{O} & -1.20246534 & 0.52393711 & 0.00000000\end{array}$

$\begin{array}{llll}\mathrm{N} & 0.00198050 & 0.57535385 & 0.00000000\end{array}$

$\begin{array}{llll}\mathrm{N} & 1.12224089 & 0.62312825 & 0.00000000\end{array}$

$\mathrm{H} \quad 0.10131394 \quad-25.53796921 \quad 0.00000000$

maug-cc-pVTZ

$-184.45653544491574$

$\begin{array}{llll}\mathrm{O} & -1.19677933 & 0.52416658 & 0.00000000\end{array}$

$\begin{array}{llll}\mathrm{N} & 0.00360602 & 0.57546801 & 0.00000000\end{array}$

$\begin{array}{llll}\mathrm{N} & 1.11493449 & 0.62292829 & 0.00000000\end{array}$

$\mathrm{H} \quad 0.10130881 \quad-25.53811288 \quad 0.00000000$

\section{CASPT2}

maug-cc-pVDZ

$-184.71461212799272$

$\begin{array}{crcc}\mathrm{O} & -1.20449497 & 0.52433752 & 0.00000000 \\ \mathrm{~N} & -0.00931927 & 0.57473905 & 0.00000000 \\ \mathrm{~N} & 1.13551742 & 0.62295729 & 0.00000000 \\ \mathrm{H} & 0.10136682 & -25.53758387 & 0.00000000\end{array}$

maug-cc-pVTZ

$-184.87379244738852$
O $\quad-1.19366856 \quad 0.52076509$
0.00000000
$\begin{array}{llll}\mathrm{N} & -0.00761981 & 0.57712564 & 0.00000000\end{array}$
$\begin{array}{llll}\mathrm{N} & 1.12351079 & 0.63083163 & 0.00000000\end{array}$
$\mathrm{H} \quad 0.10084758 \quad-25.54427236 \quad 0.00000000$ 


\section{tPBE}

maug-cc-pVDZ

$-184.94986237720883$
$\begin{array}{lll}\mathrm{O} & -1.19969123 & 0.52023315\end{array}$
0.00000000
$\begin{array}{lll}\mathrm{N} & -0.01130241 & 0.57479490\end{array}$
0.00000000
N $1.13308717 \quad 0.62730675$
0.00000000
$\mathrm{H} \quad 0.10097647 \quad-25.53788481 \quad 0.00000000$
maug-cc-pVTZ
$-184.99059461635258$

$\begin{array}{cccc}\mathrm{O} & -1.18825533 & 0.47181963 & 0.00000000 \\ \mathrm{~N} & -0.00784418 & 0.57568250 & 0.00000000 \\ \mathrm{~N} & 1.12251101 & 0.67509133 & 0.00000000 \\ \mathrm{H} & 0.09665851 & -25.53814345 & 0.00000000\end{array}$

Products

\section{CASSCF}

maug-cc-pVDZ

$-184.52572350717404$

$\begin{array}{llll}\mathrm{O} & 0.92838399 & 0.00000000 & -0.00024056\end{array}$

$\mathrm{H} \quad-0.02842918 \quad 0.00000000 \quad-0.00014628$

$\begin{array}{llll}\mathrm{N} & 1.15723292 & 0.00000000 & 30.00137474\end{array}$

$\begin{array}{llll}\mathrm{N} & 0.04281227 & 0.00000000 & 29.99901210\end{array}$

maug-cc-pVTZ

$-184.57113356074456$

$\begin{array}{llll}\mathrm{O} & 0.92546610 & 0.00000000 & -0.00032939\end{array}$

$\mathrm{H} \quad-0.02547032 \quad 0.00000000 \quad-0.00019937$

$\begin{array}{llll}\mathrm{N} & 1.15196620 & 0.00000000 & 30.00033603\end{array}$

$\mathrm{N} \quad 0.04803801 \quad 0.00000000 \quad 30.00019274$

\section{CASPT2}

maug-cc-pVDZ

$-184.82077819927684$

$\begin{array}{lrrc}\mathrm{O} & 0.93559829 & 0.00000000 & 0.01123796 \\ \mathrm{H} & -0.03602952 & 0.00000000 & -0.12140560 \\ \mathrm{~N} & 1.15620876 & 0.00000000 & 29.99694212 \\ \mathrm{~N} & 0.04422248 & 0.00000000 & 30.11322551\end{array}$

maug-cc-pVTZ

$-184.98026025453521$

$\begin{array}{rrrr}\mathrm{O} & 0.93448030 & 0.00000000 & 0.00126959 \\ \mathrm{H} & -0.03467603 & 0.00000000 & -0.00175042 \\ \mathrm{~N} & 1.15205403 & 0.00000000 & 30.00413753 \\ \mathrm{~N} & 0.04814170 & 0.00000000 & 29.99634329\end{array}$




\section{tPBE}

maug-cc-pVDZ

$-185.03120919940437$

$\begin{array}{lrrr}\mathrm{O} & 0.94566715 & 0.00000000 & -0.00038451 \\ \mathrm{H} & -0.04346261 & 0.00000000 & 0.00020008 \\ \mathrm{~N} & 1.15324129 & 0.00000000 & 29.94123755 \\ \mathrm{~N} & 0.04455418 & 0.00000000 & 30.05894687\end{array}$

maug-cc-pVTZ

$-185.07323043844292$

$\begin{array}{llll}\mathrm{O} & 0.94083454 & 0.00000000 & -0.00487167\end{array}$

$\mathrm{H} \quad-0.04086970 \quad 0.00000000 \quad 0.00458135$

$\begin{array}{llll}\mathrm{N} & 1.15149651 & 0.00000000 & 30.00526268\end{array}$

$\mathrm{N} \quad 0.04853864 \quad 0.00000000 \quad 29.99502764$

Transition state

CASSCF

maug-cc-pVDZ

$-184.35879865120182$

$\begin{array}{llll}\mathrm{O} & -0.86136380 & -0.60092904 & 0.00000000\end{array}$

$\begin{array}{llll}\mathrm{N} & 0.03703700 & 0.29934103 & 0.00000000\end{array}$

$\begin{array}{llll}\mathrm{N} & 1.13081693 & 0.60492606 & 0.00000000\end{array}$

$\mathrm{H} \quad-0.35720413 \quad-1.91439406 \quad 0.00000000$

maug-cc-pVTZ

$-184.40670267870925$

$\begin{array}{llll}\mathrm{O} & -0.86144265 & -0.60324741 & 0.00000000\end{array}$

$\begin{array}{llll}\mathrm{N} & 0.04058455 & 0.28818719 & 0.00000000\end{array}$

$\begin{array}{llll}\mathrm{N} & 1.12292359 & 0.59880682 & 0.00000000\end{array}$

$\mathrm{H} \quad-0.35277949 \quad-1.89480261 \quad 0.00000000$

\section{CASPT2}

maug-cc-pVDZ

$-184.68299250180507$

$\begin{array}{llll}\mathrm{O} & -0.86631986 & -0.57587201 & 0.00000000\end{array}$

$\begin{array}{llll}\mathrm{N} & 0.04463782 & 0.26458801 & 0.00000000\end{array}$

$\begin{array}{llll}\mathrm{N} & 1.13866575 & 0.64238567 & 0.00000000\end{array}$

$\mathrm{H} \quad-0.36769771 \quad-1.94215768 \quad 0.00000000$

maug-cc-pVTZ

$-184.84281756603374$

$\begin{array}{llll}\mathrm{O} & -0.86073454 & -0.57603391 & 0.00000000\end{array}$

$\begin{array}{llll}\mathrm{N} & 0.05127978 & 0.24921148 & 0.00000000\end{array}$

$\begin{array}{llll}\mathrm{N} & 1.12585650 & 0.63938061 & 0.00000000\end{array}$

$\mathrm{H} \quad-0.36711573 \quad-1.92361419 \quad 0.00000000$ 


\section{tPBE}

maug-cc-pVDZ

$-184.92223850941377$

$\begin{array}{cccc}\mathrm{O} & -0.84340575 & -0.57151737 & 0.00000000 \\ \mathrm{~N} & 0.06716351 & 0.24817849 & 0.00000000 \\ \mathrm{~N} & 1.13224176 & 0.69158570 & 0.00000000 \\ \mathrm{H} & -0.40671353 & -1.97930283 & 0.00000000 \\ \text { maug-cc-pVTZ } & & \\ -184.96226735784794 & \\ \mathrm{O} & -0.84125980 & -0.57333812 & 0.00000000 \\ \mathrm{~N} & 0.07127027 & 0.23868090 & 0.00000000 \\ \mathrm{~N} & 1.12272029 & 0.68475282 & 0.00000000 \\ \mathrm{H} & -0.40344475 & -1.96115161 & 0.00000000\end{array}$

R5: $\mathrm{H}+\mathrm{ClH} \rightarrow \mathrm{HCl}+\mathrm{H}$

nom-CPO

Reactants / Products

CASSCF

cc-pVDZ

maug-cc-pVDZ

$-460.60734418517637$

H $\quad 0.00000000 \quad 0.00000000 \quad-1.21390692$

$\begin{array}{llll}\mathrm{Cl} & 0.00000000 & 0.00000000 & 0.08609346\end{array}$

$\mathrm{H} \quad 0.00000000 \quad 0.00000000 \quad-32.10192155$

maug-cc-pVTZ

$-460.62323033156730$

$\mathrm{H} \quad 0.00000000 \quad 0.00000000 \quad-1.21945341$

$\begin{array}{llll}\mathrm{Cl} & 0.00000000 & 0.00000000 & 0.06977849\end{array}$

$\begin{array}{llll}\mathrm{H} & 0.00000000 & 0.00000000 & -32.08006009\end{array}$

\section{CASPT2}

maug-cc-pVDZ

$-460.74015463047101$

H $\quad 0.00000000 \quad 0.00000000 \quad-1.02905950$

$\begin{array}{llll}\mathrm{Cl} & 0.00000000 & 0.00000000 & 0.26276062\end{array}$

$\mathrm{H} \quad 0.00000000 \quad 0.00000000 \quad-32.46343613$

maug-cc-pVTZ

$-460.81082068578229$

$\mathrm{H} \quad 0.00000000 \quad 0.00000000 \quad-1.28152823$

$\begin{array}{lllll}\mathrm{Cl} & 0.00000000 & 0.00000000 & -0.00286678\end{array}$

$\mathrm{H} \quad 0.00000000 \quad 0.00000000 \quad-31.94534000$

tPBE

maug-cc-pVDZ

$-461.11060550743781$

$\mathrm{H} \quad 0.00000000 \quad 0.00000000 \quad-1.11502835$

$\begin{array}{llll}\mathrm{Cl} & 0.00000000 & 0.00000000 & 0.18448311\end{array}$

$\mathrm{H} \quad 0.00000000 \quad 0.00000000 \quad-32.29918977$ 
maug-cc-pVTZ

$-461.12637477776099$
H $\quad 0.00000000$
$0.00000000-1.48210517$
$\mathrm{Cl} \quad 0.00000000 \quad 0.00000000 \quad-0.19455534$
$\mathrm{H} \quad 0.00000000 \quad 0.00000000 \quad-31.55307450$

\section{Transition state}

CASSCF

maug-cc-pVDZ

$-460.58616945668490$

$\begin{array}{rrrr}\mathrm{H} & 0.00000000 & 0.00000000 & -1.24465349 \\ \mathrm{Cl} & 0.00000000 & 0.00000000 & 0.24746990 \\ \mathrm{H} & 0.00000000 & 0.00000000 & -2.23255142\end{array}$

maug-cc-pVTZ

$-460.60114251431031$

$\begin{array}{rrrr}\mathrm{H} & 0.00000000 & 0.00000000 & -1.24569335 \\ \mathrm{Cl} & 0.00000000 & 0.00000000 & 0.23995992 \\ \mathrm{H} & 0.00000000 & 0.00000000 & -2.22400157\end{array}$

CASPT2

maug-cc-pVDZ

$-460.72876375391132$

$\begin{array}{rrrr}\mathrm{H} & 0.00000000 & 0.00000000 & -1.22893323 \\ \mathrm{Cl} & 0.00000000 & 0.00000000 & 0.21929833 \\ \mathrm{H} & 0.00000000 & 0.00000000 & -2.22010011\end{array}$

maug-cc-pVTZ

$-460.80069438773933$

$\begin{array}{cccc}\mathrm{H} & 0.00000000 & 0.00000000 & -1.22394546 \\ \mathrm{Cl} & 0.00000000 & 0.00000000 & 0.20693441 \\ \mathrm{H} & 0.00000000 & 0.00000000 & -2.21272396\end{array}$

tPBE

maug-cc-pVDZ

$-461.10438935283958$

$\begin{array}{rrrr}\mathrm{H} & 0.00000000 & 0.00000000 & -1.16539172 \\ \mathrm{Cl} & 0.00000000 & 0.00000000 & 0.22708895 \\ \mathrm{H} & 0.00000000 & 0.00000000 & -2.29143224\end{array}$

maug-cc-pVTZ

$-461.11893494666623$

$\begin{array}{rrrr}\mathrm{H} & 0.00000000 & 0.00000000 & -1.16904002 \\ \mathrm{Cl} & 0.00000000 & 0.00000000 & 0.21666370 \\ \mathrm{H} & 0.00000000 & 0.00000000 & -2.27735870\end{array}$ 


\section{mod-CPO}

Reactants / Products

CASSCF

maug-cc-pVDZ

$-460.64014441975866$

$\mathrm{H} \quad 0.00000000 \quad 0.00000000 \quad-1.20542000$

$\begin{array}{llll}\mathrm{Cl} & 0.00000000 & 0.00000000 & 0.07600263\end{array}$

$\mathrm{H} \quad 0.00000000 \quad 0.00000000 \quad-32.10031764$

maug-cc-pVTZ

$-460.66223418303053$

H $\quad 0.00000000 \quad 0.00000000 \quad-1.20840079$

$\begin{array}{llll}\mathrm{Cl} & 0.00000000 & 0.00000000 & 0.06358413\end{array}$

$\mathrm{H} \quad 0.00000000 \quad 0.00000000 \quad-32.08491835$

\section{CASPT2}

maug-cc-pVDZ

$-460.74251808464857$

$\begin{array}{cccc}\mathrm{H} & 0.00000000 & 0.00000000 & -1.08522950 \\ \mathrm{Cl} & 0.00000000 & 0.00000000 & 0.19935687 \\ \mathrm{H} & 0.00000000 & 0.00000000 & -32.34386238 \\ \text { maug-cc-pVTZ } & & \\ -460.81493560073034 \\ \mathrm{H} & 0.00000000 & 0.00000000 & -1.21450521 \\ \mathrm{Cl} & 0.00000000 & 0.00000000 & 0.06114841 \\ \mathrm{H} & 0.00000000 & 0.00000000 & -32.07637822\end{array}$

tPBE

maug-cc-pVDZ

$-461.09770973825630$

$\mathrm{H} \quad 0.00000000 \quad 0.00000000 \quad-1.21696008$

$\begin{array}{llll}\mathrm{Cl} & 0.00000000 & 0.00000000 & 0.09114126\end{array}$

$\mathrm{H} \quad 0.00000000 \quad 0.00000000 \quad-32.10391619$

maug-cc-pVTZ

$-461.11131287263862$

H $\quad 0.00000000 \quad 0.00000000 \quad-1.22007855$

$\begin{array}{llll}\mathrm{Cl} & 0.00000000 & 0.00000000 & 0.07535373\end{array}$

$\mathrm{H} \quad 0.00000000 \quad 0.00000000 \quad-32.08501019$

Transition state

CASSCF

maug-cc-pVDZ

$-460.61006962260035$

H $\quad 0.00000000 \quad 0.00000000 \quad-1.30818150$

$\begin{array}{llll}\mathrm{Cl} & 0.00000000 & 0.00000000 & 0.27038785\end{array}$

$\mathrm{H} \quad 0.00000000 \quad 0.00000000 \quad-2.19194136$ 
maug-cc-pVTZ

$-460.63036390144862$

$\begin{array}{rrrr}\mathrm{H} & 0.00000000 & 0.00000000 & -1.32235219 \\ \mathrm{Cl} & 0.00000000 & 0.00000000 & 0.26698470 \\ \mathrm{H} & 0.00000000 & 0.00000000 & -2.17436752\end{array}$

\section{CASPT2}

maug-cc-pVDZ

$-460.73020120351362$

$\begin{array}{cccc}\mathrm{H} & 0.00000000 & 0.00000000 & -1.23114603 \\ \mathrm{Cl} & 0.00000000 & 0.00000000 & 0.21841277 \\ \mathrm{H} & 0.00000000 & 0.00000000 & -2.21700174 \\ \text { maug-cc-pVTZ } & & \\ -460.80334214904957 \\ \mathrm{H} & 0.00000000 & 0.00000000 & -1.23175478 \\ \mathrm{Cl} & 0.00000000 & 0.00000000 & 0.20508731 \\ \mathrm{H} & 0.00000000 & 0.00000000 & -2.20306754\end{array}$

\section{tPBE}

maug-cc-pVDZ

$-461.09289268713377$

$\begin{array}{rrrr}\mathrm{H} & 0.00000000 & 0.00000000 & -1.13438062 \\ \mathrm{Cl} & 0.00000000 & 0.00000000 & 0.24714967 \\ \mathrm{H} & 0.00000000 & 0.00000000 & -2.34250407\end{array}$

maug-cc-pVTZ

$-461.10532404050508$

$\begin{array}{rrrr}\mathrm{H} & 0.00000000 & 0.00000000 & -1.14102810 \\ \mathrm{Cl} & 0.00000000 & 0.00000000 & 0.23360205 \\ \mathrm{H} & 0.00000000 & 0.00000000 & -2.32230896\end{array}$

R6: $\mathrm{CH}_{3}+\mathrm{FCl} \rightarrow \mathrm{CH}_{3} \mathrm{~F}+\mathrm{Cl}$

nom-CPO

Reactants

CASSCF

maug-cc-pVDZ

$-598.45139886050708$

Cl $\quad-1.97948362 \quad-0.83552869 \quad-31.02294073$

F $\quad-2.10240901 \quad-0.78134179 \quad-29.31176249$

$\begin{array}{llll}\text { C } & -5.84825198 & 0.88000262 & 22.48388639\end{array}$

$\mathrm{H} \quad-6.92806338 \quad 0.87999985 \quad 22.48266782$

$\mathrm{H} \quad-5.30834362 \quad-0.05514057 \quad 22.48292405$

$\mathrm{H} \quad-5.30834840 \quad 1.81514857 \quad 22.48292496$ 


\begin{tabular}{|c|c|c|c|}
\hline \multicolumn{4}{|c|}{$\begin{array}{l}\text { naug-cc-pVTZ } \\
-598.51156292167002\end{array}$} \\
\hline $\mathrm{Cl}$ & -1.98108668 & -0.83496725 & -30 \\
\hline $\mathrm{F}$ & -2.10081372 & -0.78189256 & -29.33927481 \\
\hline $\mathrm{C}$ & -5.84824984 & 0.87999995 & 22.48312124 \\
\hline $\mathrm{H}$ & -6.91800992 & 0.87999997 & 22.48309306 \\
\hline $\mathrm{H}$ & -5.31336995 & -0.04643843 & 22.48308930 \\
\hline $\mathrm{H}$ & -5.31336990 & 1.80643831 & 22.4830899 \\
\hline
\end{tabular}

\section{CASPT2}

maug-cc-pVDZ

$\begin{array}{cccc}\mathrm{Cl} & -1.97827709 & -0.83708789 & -31.02178188 \\ \mathrm{~F} & -2.10230217 & -0.78032996 & -29.33186351 \\ \mathrm{C} & -5.84856383 & 0.88013956 & 22.48784429 \\ \mathrm{H} & -6.93857135 & 0.88013896 & 22.48718355 \\ \mathrm{H} & -5.30359284 & -0.06380945 & 22.48840332 \\ \mathrm{H} & -5.30359273 & 1.82408878 & 22.48791422 \\ \text { maug-cc-pVTZ } & & \\ -599.08337701944777 & \\ \mathrm{Cl} & -1.98237826 & -0.83437271 & -30.97770694 \\ \mathrm{~F} & -2.10069115 & -0.78196929 & -29.34077854 \\ \mathrm{C} & -5.84794012 & 0.87987018 & 22.47899265 \\ \mathrm{H} & -6.92284013 & 0.87987111 & 22.47915239 \\ \mathrm{H} & -5.31052491 & -0.05099620 & 22.47898889 \\ \mathrm{H} & -5.31052542 & 1.81073692 & 22.47905156\end{array}$

tPBE

maug-cc-pVDZ

$-599.43089499150972$

$\begin{array}{cccc}\mathrm{Cl} & -1.98080272 & -0.83509339 & -30.99934464 \\ \mathrm{~F} & -2.10109668 & -0.78176691 & -29.33535779 \\ \mathrm{C} & -5.84824804 & 0.87999989 & 22.48310754 \\ \mathrm{H} & -6.94434717 & 0.88000075 & 22.48311174 \\ \mathrm{H} & -5.30020206 & -0.06925099 & 22.48308498 \\ \mathrm{H} & -5.30020332 & 1.82925066 & 22.48309817 \\ \text { maug-cc-pVTZ } & & \\ -599.48890735762723 & \\ \mathrm{Cl} & -1.98192904 & -0.83459827 & -30.98345428 \\ \mathrm{~F} & -2.09994985 & -0.78227372 & -29.35124300 \\ \mathrm{C} & -5.84825251 & 0.88000279 & 22.48294360 \\ \mathrm{H} & -6.93280176 & 0.88000035 & 22.48385344 \\ \mathrm{H} & -5.30598024 & -0.05924440 & 22.48245396 \\ \mathrm{H} & -5.30598660 & 1.81925325 & 22.48314629\end{array}$




\section{Products}

\section{CASSCF}

maug-cc-pVDZ

$-598.53817999274099$

$\begin{array}{cccc}\mathrm{Cl} & 24.67591084 & -0.07839511 & -0.00190602 \\ \mathrm{~F} & -0.73389430 & 0.00656900 & -0.00135121 \\ \mathrm{C} & -2.10366763 & -0.00731699 & 0.00254506 \\ \mathrm{H} & -2.44817465 & -0.82759553 & -0.62487427 \\ \mathrm{H} & -2.45048366 & -0.13665645 & 1.02635491 \\ \mathrm{H} & -2.46949060 & 0.96023509 & -0.40282847\end{array}$

maug-cc-pVTZ

$-598.59368945941594$

$\begin{array}{cccc}\mathrm{Cl} & 24.67586043 & -0.07847066 & -0.00187722 \\ \mathrm{~F} & -0.74089894 & 0.00739333 & -0.00168272 \\ \mathrm{C} & -2.10105663 & -0.00693194 & 0.00238711 \\ \mathrm{H} & -2.44652232 & -0.82077403 & -0.61933948 \\ \mathrm{H} & -2.44884156 & -0.13581678 & 1.01760378 \\ \mathrm{H} & -2.46834098 & 0.95144008 & -0.39915147\end{array}$

\section{CASPT2}

maug-cc-pVDZ

$$
-598.98000914740533
$$

$\begin{array}{cccc}\mathrm{Cl} & 24.67590937 & -0.07796834 & -0.00208134 \\ \mathrm{~F} & -0.71219196 & 0.00470153 & -0.00056693 \\ \mathrm{C} & -2.10846373 & -0.00257744 & 0.00055917 \\ \mathrm{H} & -2.45726225 & -0.83299399 & -0.63096645 \\ \mathrm{H} & -2.45955699 & -0.13609108 & 1.03446717 \\ \mathrm{H} & -2.46823443 & 0.96176931 & -0.40347162\end{array}$

maug-cc-pVTZ

$-599.17957660425827$
Cl $24.67513489 \quad-0.07801950 \quad-0.00206258$
F $\quad-0.72266956 \quad 0.00524513 \quad-0.00077736$
C $\quad-2.10755734 \quad-0.00242745 \quad 0.00049423$
$\mathrm{H} \quad-2.45372568 \quad-0.82194632 \quad-0.62241549$
$\begin{array}{llll}\mathrm{H} & -2.45602259 & -0.13443117 & 1.02059000\end{array}$
$\mathrm{H} \quad-2.46495973 \quad 0.94841932 \quad-0.39788879$

\section{tPBE}

maug-cc-pVDZ

$-599.52789984550077$
$\mathrm{Cl} \quad 24.67584625 \quad-0.07885651 \quad-0.00172906$
F $\quad-0.70907496 \quad 0.01395897 \quad-0.00426008$
C $\quad-2.10563719 \quad-0.00066152 \quad-0.00024261$
$\mathrm{H} \quad-2.45659821 \quad-0.83942996 \quad-0.63302095$
$\begin{array}{llll}\mathrm{H} & -2.45913061 & -0.13922261 & 1.04040849\end{array}$
H $\quad-2.47520527 \quad 0.96105163 \quad-0.40321579$ 
maug-cc-pVTZ

$-599.57818943303243$

$\begin{array}{cccc}\mathrm{Cl} & 24.67746948 & -0.07846166 & -0.00187050 \\ \mathrm{~F} & -0.71490803 & 0.01008708 & -0.00288541 \\ \mathrm{C} & -2.10621405 & -0.00060982 & -0.00027347 \\ \mathrm{H} & -2.45658053 & -0.83015493 & -0.62721113 \\ \mathrm{H} & -2.45879769 & -0.13676017 & 1.02982412 \\ \mathrm{H} & -2.47076919 & 0.95273951 & -0.39964361\end{array}$

\section{Transition state}

\section{CASSCF}

maug-cc-pVDZ

$-598.43603125290804$

$\begin{array}{crrr}\mathrm{Cl} & 1.60020368 & -0.00020955 & 0.00000000 \\ \mathrm{~F} & -0.29603563 & -0.00014946 & 0.00000000 \\ \mathrm{C} & -2.44475140 & 0.00023548 & 0.00000000 \\ \mathrm{H} & -2.58368826 & -0.53552395 & 0.92800121 \\ \mathrm{H} & -2.58368826 & -0.53552395 & -0.92800121 \\ \mathrm{H} & -2.58340466 & 1.07182419 & 0.00000000\end{array}$

maug-cc-pVTZ

$-598.48807901748967$

$\begin{array}{crrc}\mathrm{Cl} & 1.55777065 & -0.00102420 & 0.00000000 \\ \mathrm{~F} & -0.32605926 & 0.00017370 & 0.00000000 \\ \mathrm{C} & -2.42069944 & 0.00047653 & 0.00000000 \\ \mathrm{H} & -2.56692160 & -0.52990748 & 0.91842436 \\ \mathrm{H} & -2.56692160 & -0.52990748 & -0.91842436 \\ \mathrm{H} & -2.56853328 & 1.06084171 & 0.00000000\end{array}$

\section{CASPT2}

maug-cc-pVDZ

$-598.87159706093473$

$\begin{array}{cccc}\mathrm{Cl} & 1.52126868 & -0.00035729 & 0.00000000 \\ \mathrm{~F} & -0.27644533 & -0.00009959 & 0.00000000 \\ \mathrm{C} & -2.46358232 & 0.00024931 & 0.00000000 \\ \mathrm{H} & -2.55762164 & -0.54285442 & 0.94074356 \\ \mathrm{H} & -2.55762164 & -0.54285442 & -0.94074356 \\ \mathrm{H} & -2.55736226 & 1.08656920 & 0.00000000\end{array}$

maug-cc-pVTZ

$-599.07278219723742$

$\begin{array}{crrr}\text { Cl } & 1.44408485 & -0.00037676 & 0.00000000 \\ \text { F } & -0.32692197 & -0.00007112 & 0.00000000 \\ \text { C } & -2.42167316 & 0.00024259 & 0.00000000 \\ \text { H } & -2.52903483 & -0.53472057 & 0.92666053 \\ \text { H } & -2.52903483 & -0.53472057 & -0.92666053 \\ \text { H } & -2.52878460 & 1.07029923 & 0.00000000\end{array}$




\section{tPBE}

maug-cc-pVDZ $-599.42511782929193$

$\begin{array}{crrc}\mathrm{Cl} & 1.47984184 & -0.00070540 & 0.00000000 \\ \mathrm{~F} & -0.26900421 & -0.00012276 & 0.00000000 \\ \mathrm{C} & -2.46584395 & 0.00036517 & 0.00000000 \\ \mathrm{H} & -2.54556888 & -0.54586526 & 0.94609860 \\ \mathrm{H} & -2.54556888 & -0.54586526 & -0.94609860 \\ \mathrm{H} & -2.54522045 & 1.09284630 & 0.00000000\end{array}$
maug-cc-pVTZ

$-599.47791800356015$

$\begin{array}{crrr}\mathrm{Cl} & 1.42515297 & -0.00096217 & 0.00000000 \\ \mathrm{~F} & -0.31785879 & 0.00053875 & 0.00000000 \\ \mathrm{C} & -2.43030312 & 0.00028450 & 0.00000000 \\ \mathrm{H} & -2.52257826 & -0.53960298 & 0.93509082 \\ \mathrm{H} & -2.52257826 & -0.53960298 & -0.93509082 \\ \mathrm{H} & -2.52319905 & 1.07999768 & 0.00000000\end{array}$

\section{mod-CPO}

\section{Reactants}

\section{CASSCF}

maug-cc-pVDZ

$-598.54786080844099$

$\begin{array}{cccc}\mathrm{Cl} & -1.97531229 & -0.84110132 & -31.00302674 \\ \mathrm{~F} & -2.10669940 & -0.77559436 & -29.33246803 \\ \mathrm{C} & -5.84822217 & 0.87995921 & 22.48330036 \\ \mathrm{H} & -6.92803181 & 0.87996124 & 22.48327525 \\ \mathrm{H} & -5.30831988 & -0.05518457 & 22.48330595 \\ \mathrm{H} & -5.30831445 & 1.81509979 & 22.48331322 \\ \text { maug-cc-pVTZ } & & \\ -598.61796566875182 & \\ \mathrm{Cl} & -1.98563419 & -0.83053287 & -30.96659587 \\ \mathrm{~F} & -2.09822507 & -0.78552071 & -29.33959868 \\ \mathrm{C} & -5.84776075 & 0.87979886 & 22.47597455 \\ \mathrm{H} & -6.91751441 & 0.87981317 & 22.47677550 \\ \mathrm{H} & -5.31289701 & -0.04664281 & 22.47563550 \\ \mathrm{H} & -5.31286856 & 1.80622437 & 22.47550900\end{array}$

\section{CASPT2}

maug-cc-pVDZ

$\begin{array}{cccc}\mathrm{Cl} & -1.96492226 & -0.84213289 & -1.17556449 \\ \mathrm{~F} & -2.11697774 & -0.77472711 & 0.51426449 \\ \mathrm{C} & -5.84823169 & 0.88000000 & 22.48310000 \\ \mathrm{H} & -6.93822060 & 0.88000000 & 22.48310000 \\ \mathrm{H} & -5.30327385 & -0.06393337 & 22.48310000 \\ \mathrm{H} & -5.30327385 & 1.82393337 & 22.48310000\end{array}$




$\begin{array}{cccc}\text { maug-cc-pVTZ } & & \\ \text { Cl } & -1.96730947 & -0.84107465 & -1.14903483 \\ \text { F } & -2.11459053 & -0.77578535 & 0.48773483 \\ \text { C } & -5.84822903 & 0.88000000 & 22.48310000 \\ \text { H } & -6.92310920 & 0.88000000 & 22.48310000 \\ \text { H } & -5.31083088 & -0.05085151 & 22.48310000 \\ \text { H } & -5.31083088 & 1.81085151 & 22.48310000\end{array}$

\section{tPBE}

maug-cc-pVDZ

$-599.40558363375965$

$\begin{array}{cccc}\mathrm{Cl} & -1.97902676 & -0.83591161 & -31.02342505 \\ \mathrm{~F} & -2.10141558 & -0.78160224 & -29.33138005 \\ \mathrm{C} & -5.84861191 & 0.88016346 & 22.48812181 \\ \mathrm{H} & -6.94471932 & 0.88015372 & 22.48826908 \\ \mathrm{H} & -5.30055500 & -0.06908957 & 22.48777252 \\ \mathrm{H} & -5.30057143 & 1.82942624 & 22.48834170 \\ \text { maug-cc-pVTZ } & & \\ -599.46025205241926 & \\ \mathrm{Cl} & -1.98110142 & -0.83495548 & -30.99482381 \\ \mathrm{~F} & -2.10094374 & -0.78184659 & -29.33720584 \\ \mathrm{C} & -5.84821313 & 0.87998431 & 22.48241968 \\ \mathrm{H} & -6.93276371 & 0.87997946 & 22.48399172 \\ \mathrm{H} & -5.30593207 & -0.05926031 & 22.48088862 \\ \mathrm{H} & -5.30594593 & 1.81923862 & 22.48242963\end{array}$

\section{Products}

CASSCF

maug-cc-pVDZ

$-598.64443604540679$

$\begin{array}{cccc}\mathrm{Cl} & 24.67574011 & -0.08243128 & -0.00788769 \\ \mathrm{~F} & -0.71180978 & 0.04723253 & 0.05315931 \\ \mathrm{C} & -2.11939600 & -0.00294651 & -0.00190074 \\ \mathrm{H} & -2.40437539 & -0.83688703 & -0.64044398 \\ \mathrm{H} & -2.49350693 & -0.14663143 & 1.00992643 \\ \mathrm{H} & -2.47645200 & 0.93850371 & -0.41491332\end{array}$

maug-cc-pVTZ

$-598.70732930318900$
$\mathrm{Cl} \quad 24.67584817 \quad-0.07779385 \quad-0.00217803$
F $\quad-0.71961922 \quad 0.00396488 \quad-0.00005131$
C $\quad-2.11566832 \quad-0.00135718 \quad 0.00002812$
$\mathrm{H} \quad-2.45371580 \quad-0.81798985 \quad-0.62090868$
$\mathrm{H} \quad-2.45623486 \quad-0.13275357 \quad 1.01662870$
$\mathrm{H} \quad-2.46040997 \quad 0.94276958 \quad-0.39557879$ 


\section{CASPT2}

maug-cc-pVDZ

$\begin{array}{cccc}\mathrm{Cl} & 24.67574011 & -0.08243128 & -0.00788769 \\ \mathrm{~F} & -0.71258110 & 0.00442990 & 0.00082180 \\ \mathrm{C} & -2.10713999 & -0.00123434 & 0.00015087 \\ \mathrm{H} & -2.45984828 & -0.83073165 & -0.63120090 \\ \mathrm{H} & -2.46055916 & -0.13570971 & 1.03358935 \\ \mathrm{H} & -2.46567146 & 0.95788580 & -0.40324113 \\ \text { maug-cc-pVTZ } & & \\ \mathrm{Cl} & 24.67574011 & -0.08243128 & -0.00788769 \\ \mathrm{~F} & -0.72212964 & 0.00325352 & -0.00031832 \\ \mathrm{C} & -2.10628677 & -0.00083137 & 0.00029319 \\ \mathrm{H} & -2.45524116 & -0.82349967 & -0.62506311 \\ \mathrm{H} & -2.45874341 & -0.13415588 & 1.02418974 \\ \mathrm{H} & -2.46339901 & 0.94987339 & -0.39898149\end{array}$

TPBE

maug-cc-pVDZ

$-599.49299187635756$

$\begin{array}{cccc}\text { Cl } & 24.67555983 & -0.07725955 & -0.00124817 \\ \text { F } & -0.70687153 & -0.00089935 & -0.00860734 \\ \text { C } & -2.10569648 & -0.00117065 & 0.00026480 \\ \text { H } & -2.46807877 & -0.83486750 & -0.63239802 \\ \text { H } & -2.45766404 & -0.13433417 & 1.04186881 \\ \text { H } & -2.46704901 & 0.96537123 & -0.40194009 \\ \text { maug-cc-pVTZ } & & \\ -599.54076090276180 & \\ \text { Cl } & 24.67565332 & -0.07768900 & -0.00209195 \\ \text { F } & -0.71262033 & 0.00302046 & -0.00084843 \\ \text { C } & -2.10594670 & -0.00128836 & 0.00004930 \\ \text { H } & -2.46013720 & -0.82785657 & -0.62839365 \\ \text { H } & -2.46122190 & -0.13411387 & 1.02948076 \\ \text { H } & -2.46552719 & 0.95476734 & -0.40025602\end{array}$

Transition state

CASSCF

maug-cc-pVDZ

$-598.52769267945303$

$\begin{array}{cccc}\mathrm{Cl} & 1.52659251 & -0.00069016 & 0.00000000 \\ \mathrm{~F} & -0.32145951 & 0.00019580 & 0.00000000 \\ \mathrm{C} & -2.41853945 & 0.00032266 & 0.00000000 \\ \mathrm{H} & -2.55919392 & -0.53521607 & 0.92747545 \\ \mathrm{H} & -2.55919392 & -0.53521607 & -0.92747545 \\ \mathrm{H} & -2.55957024 & 1.07125663 & 0.00000000\end{array}$


maug-cc-pVTZ

$-598.58746509415607$

$\begin{array}{crrr}\text { Cl } & 1.48691550 & -0.00053406 & 0.00000000 \\ \text { F } & -0.35285516 & 0.00002298 & 0.00000000 \\ \text { C } & -2.39428969 & 0.00030393 & 0.00000000 \\ \text { H } & -2.54367025 & -0.52961718 & 0.91781973 \\ \text { H } & -2.54367025 & -0.52961718 & -0.91781973 \\ \text { H } & -2.54379469 & 1.06009430 & 0.00000000\end{array}$

\section{CASPT2}

maug-cc-pVDZ

$-598.87306063407777$

$\begin{array}{crrr}\mathrm{Cl} & 1.52965274 & -0.00020422 & 0.00000000 \\ \mathrm{~F} & -0.26886595 & -0.00032315 & 0.00000000 \\ \mathrm{C} & -2.47307054 & 0.00026323 & 0.00000000 \\ \mathrm{H} & -2.55982318 & -0.54313423 & 0.94127165 \\ \mathrm{H} & -2.55982318 & -0.54313423 & -0.94127165 \\ \mathrm{H} & -2.55943442 & 1.08718540 & 0.00000000\end{array}$

maug-cc-pVTZ

$-599.07324847432028$

$\begin{array}{crrc}\mathrm{Cl} & 1.45384313 & -0.00032167 & 0.00000000 \\ \mathrm{~F} & -0.32014443 & -0.00017220 & 0.00000000 \\ \mathrm{C} & -2.42844105 & 0.00023971 & 0.00000000 \\ \mathrm{H} & -2.53228723 & -0.53474391 & 0.92676800 \\ \mathrm{H} & -2.53228723 & -0.53474391 & -0.92676800 \\ \mathrm{H} & -2.53204772 & 1.07039479 & 0.00000000\end{array}$

tPBE

maug-cc-pVDZ

$-599.40272734153041$

$\begin{array}{cccc}\mathrm{Cl} & 1.56140250 & -0.00033599 & 0.00000000 \\ \mathrm{~F} & -0.20662962 & -0.00014960 & 0.00000000 \\ \mathrm{C} & -2.51559737 & 0.00028576 & 0.00000000 \\ \mathrm{H} & -2.57693815 & -0.54661345 & 0.94725062 \\ \mathrm{H} & -2.57693815 & -0.54661345 & -0.94725062 \\ \mathrm{H} & -2.57666374 & 1.09407953 & 0.00000000 \\ \text { maug-cc-pVTZ } & & \\ -599.45298612079307 & \\ \mathrm{Cl} & 1.49384937 & -0.00053767 & 0.00000000 \\ \mathrm{~F} & -0.27137163 & 0.00005980 & 0.00000000 \\ \mathrm{C} & -2.47155561 & 0.00028553 & 0.00000000 \\ \mathrm{H} & -2.54740920 & -0.54027695 & 0.93627241 \\ \mathrm{H} & -2.54740920 & -0.54027695 & -0.93627241 \\ \mathrm{H} & -2.54746824 & 1.08139903 & 0.00000000\end{array}$




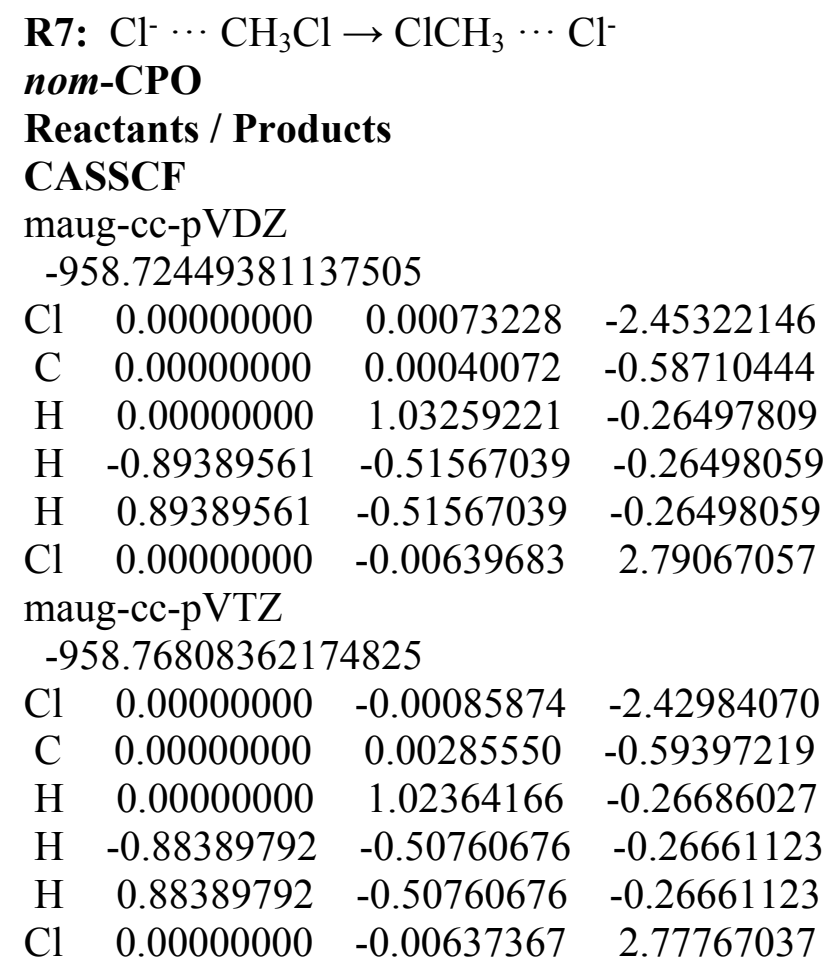

\section{CASPT2}

maug-cc-pVDZ

$-959.12817713769334$

$\begin{array}{rrrr}\mathrm{Cl} & 0.00000000 & -0.00106738 & -2.39950701 \\ \mathrm{C} & 0.00000000 & -0.00162456 & -0.57232083 \\ \mathrm{H} & 0.00000000 & 1.03531310 & -0.22461948 \\ \mathrm{H} & -0.89808608 & -0.52026069 & -0.22515566 \\ \mathrm{H} & 0.89808608 & -0.52026069 & -0.22515566 \\ \mathrm{Cl} & 0.00000000 & -0.00070248 & 2.64198897\end{array}$

maug-cc-pVTZ

$-959.31024198092234$

$\begin{array}{llll}\mathrm{Cl} & 0.00000000 & 0.00057828 & -2.37745442\end{array}$

C $\quad 0.00000000 \quad-0.00070492 \quad-0.56211159$

$\mathrm{H} \quad 0.00000000 \quad 1.02217788 \quad-0.21699920$

$\mathrm{H} \quad-0.88607184 \quad-0.51254117 \quad-0.21836270$

$\mathrm{H} \quad 0.88607184 \quad-0.51254117 \quad-0.21836270$

$\begin{array}{llll}\mathrm{Cl} & 0.00000000 & 0.00214792 & 2.59531390\end{array}$ 


\section{tPBE}

maug-cc-pVDZ

$-959.94832686421955$

$\begin{array}{rrrr}\mathrm{Cl} & 0.00000000 & -0.00215081 & -2.40948826 \\ \mathrm{C} & 0.00000000 & -0.00165021 & -0.58108491 \\ \mathrm{H} & 0.00000000 & 1.04063407 & -0.23014515 \\ \mathrm{H} & -0.90253255 & -0.52263329 & -0.22962866 \\ \mathrm{H} & 0.90253255 & -0.52263329 & -0.22962866 \\ \mathrm{Cl} & 0.00000000 & -0.00254177 & 2.67073296\end{array}$

maug-cc-pVTZ

$-959.97769504613757$

$\begin{array}{rrrr}\mathrm{Cl} & 0.00000000 & -0.00032744 & -2.40849349 \\ \mathrm{C} & 0.00000000 & -0.00093769 & -0.57932120 \\ \mathrm{H} & 0.00000000 & 1.03099045 & -0.23154667 \\ \mathrm{H} & -0.89342863 & -0.51649907 & -0.23053999 \\ \mathrm{H} & 0.89342863 & -0.51649907 & -0.23053999 \\ \mathrm{Cl} & 0.00000000 & -0.00156826 & 2.67028735\end{array}$

\section{Transition state}

\section{CASSCF}

maug-cc-pVDZ

$-958.69296385005237$

$\begin{array}{rrrc}\mathrm{Cl} & 2.39161140 & 0.00024064 & 0.00000000 \\ \mathrm{Cl} & -2.39161140 & 0.00024064 & 0.00000000 \\ \mathrm{C} & 0.00000000 & 0.00006220 & 0.00000000 \\ \mathrm{H} & 0.00000000 & -0.53611644 & -0.92643946 \\ \mathrm{H} & 0.00000000 & -0.53611644 & 0.92643946 \\ \mathrm{H} & 0.00000000 & 1.07040926 & 0.00000000\end{array}$

maug-cc-pVTZ

$-958.73098096977412$

$\begin{array}{cccc}\text { Cl } & 2.38055568 & 0.00156827 & 0.00000000 \\ \mathrm{Cl} & -2.38055568 & 0.00156827 & 0.00000000 \\ \mathrm{C} & 0.00000000 & 0.00138333 & 0.00000000 \\ \mathrm{H} & 0.00000000 & -0.52943365 & -0.91724086 \\ \mathrm{H} & 0.00000000 & -0.52943365 & 0.91724086 \\ \mathrm{H} & 0.00000000 & 1.06107773 & 0.00000000\end{array}$

\section{CASPT2}

maug-cc-pVDZ

$-959.10299470787879$
Cl $2.31251751 \quad-0.00153811$
$\begin{array}{rrrr}\mathrm{Cl} & -2.31251751 & -0.00153811 & 0.00000000 \\ \mathrm{C} & 0.00000000 & -0.00166848 & 0.00000000\end{array}$
$\mathrm{H} \quad 0.00000000 \quad-0.54376036 \quad-0.93782619$
$\mathrm{H} \quad 0.00000000 \quad-0.54376036 \quad 0.93782619$
$\mathrm{H} \quad 0.00000000 \quad 1.08156261 \quad 0.00000000$ 


\begin{tabular}{cccc}
\multicolumn{4}{c}{ maug-cc-pVTZ } \\
-959.28799383629359 & \\
$\mathrm{Cl}$ & 2.28382497 & 0.00015231 & 0.00000000 \\
$\mathrm{Cl}$ & -2.28382497 & 0.00015231 & 0.00000000 \\
$\mathrm{C}$ & 0.00000000 & 0.00007984 & 0.00000000 \\
$\mathrm{H}$ & 0.00000000 & -0.53435080 & -0.92537024 \\
$\mathrm{H}$ & 0.00000000 & -0.53435080 & 0.92537024 \\
$\mathrm{H}$ & 0.00000000 & 1.06871432 & 0.00000000
\end{tabular}

\section{tPBE}

maug-cc-pVDZ

$$
-959.92920829302204
$$

$\begin{array}{rrrr}\mathrm{Cl} & 2.31835931 & -0.00236695 & 0.00000000 \\ \mathrm{Cl} & -2.31835931 & -0.00236695 & 0.00000000 \\ \mathrm{C} & 0.00000000 & -0.00245297 & 0.00000000 \\ \mathrm{H} & 0.00000000 & -0.54792097 & -0.94349992 \\ \mathrm{H} & 0.00000000 & -0.54792097 & 0.94349992 \\ \mathrm{H} & 0.00000000 & 1.08733655 & 0.00000000\end{array}$

maug-cc-pVTZ

$-959.96488312956069$
$\mathrm{Cl} \quad 2.30522328 \quad-0.00089305$
$\mathrm{Cl}-2.30522328 \quad-0.00089305$
0.00000000
$\begin{array}{lll}\text { C } & 0.00000000 & -0.00097275\end{array}$
0.00000000
$\mathrm{H} \quad 0.00000000 \quad-0.54009274$
0.00000000
$\mathrm{H} \quad 0.00000000 \quad-0.54009274$
$-0.93299294$
H $\quad 0.00000000 \quad 1.07655421$
0.93299294
0.00000000

\section{mod-CPO}

\section{Reactants / Products}

\section{CASSCF}

maug-cc-pVDZ

$-958.78625444439308$

$\begin{array}{cccc}\mathrm{Cl} & 0.00000000 & 0.00355940 & -2.41946632 \\ \mathrm{C} & 0.00000000 & -0.00122991 & -0.58331261 \\ \mathrm{H} & 0.00000000 & 1.02672232 & -0.24847464 \\ \mathrm{H} & -0.89109991 & -0.51663503 & -0.25290843 \\ \mathrm{H} & 0.89109991 & -0.51663503 & -0.25290843 \\ \mathrm{Cl} & 0.00000000 & -0.00075879 & 2.72454798 \\ \text { maug-cc-pVTZ } & & \\ -958.83779209434419 & \\ \mathrm{Cl} & 0.00000000 & 0.00290322 & -2.41998749 \\ \mathrm{C} & 0.00000000 & 0.00106820 & -0.59110879 \\ \mathrm{H} & 0.00000000 & 1.02075857 & -0.26021504 \\ \mathrm{H} & -0.88338365 & -0.50930432 & -0.26182853 \\ \mathrm{H} & 0.88338365 & -0.50930432 & -0.26182853 \\ \mathrm{Cl} & 0.00000000 & -0.00376769 & 2.75352585\end{array}$




\section{CASPT2}

maug-cc-pVDZ

$-959.12939462308600$

$\begin{array}{crrr}\mathrm{Cl} & 0.00000000 & -0.00120773 & -2.40081651 \\ \mathrm{C} & 0.00000000 & -0.00133249 & -0.57777984 \\ \mathrm{H} & 0.00000000 & 1.03540314 & -0.22824233 \\ \mathrm{H} & -0.89783686 & -0.51970321 & -0.22835239 \\ \mathrm{H} & 0.89783686 & -0.51970321 & -0.22835239 \\ \mathrm{Cl} & 0.00000000 & -0.00150171 & 2.65557705\end{array}$

maug-cc-pVTZ

$-959.31391122241473$

$\begin{array}{rrrr}\mathrm{Cl} & 0.00000000 & 0.00038369 & -2.38011066 \\ \mathrm{C} & 0.00000000 & -0.00009907 & -0.56948524 \\ \mathrm{H} & 0.00000000 & 1.02236088 & -0.22242974 \\ \mathrm{H} & -0.88549998 & -0.51139791 & -0.22284541 \\ \mathrm{H} & 0.88549998 & -0.51139791 & -0.22284541 \\ \mathrm{Cl} & 0.00000000 & 0.00041039 & 2.61525703\end{array}$

\section{tPBE}

maug-cc-pVDZ

$-959.92512571099542$

\begin{tabular}{crrr}
$\mathrm{Cl}$ & 0.00000000 & 0.01435469 & -2.48040610 \\
$\mathrm{C}$ & 0.00000000 & -0.00601439 & -0.65120220 \\
$\mathrm{H}$ & 0.00000000 & 1.03295067 & -0.28718677 \\
$\mathrm{H}$ & -0.90354472 & -0.53154627 & -0.30572704 \\
$\mathrm{H}$ & 0.90354472 & -0.53154627 & -0.30572704 \\
$\mathrm{Cl}$ & 0.00000000 & 0.00191328 & 2.94490811 \\
maug-cc-pVTZ & & \\
\multicolumn{4}{c}{-959.95624778808633} \\
$\mathrm{Cl}$ & 0.00000000 & 0.00052485 & -2.40401583 \\
$\mathrm{C}$ & 0.00000000 & -0.00080108 & -0.58738841 \\
$\mathrm{H}$ & 0.00000000 & 1.02950862 & -0.23559134 \\
$\mathrm{H}$ & -0.89250624 & -0.51632142 & -0.23671705 \\
$\mathrm{H}$ & 0.89250624 & -0.51632142 & -0.23671705 \\
$\mathrm{Cl}$ & 0.00000000 & -0.00125298 & 2.68409862
\end{tabular}

Transition state

CASSCF

maug-cc-pVDZ

$-958.75967418717266$

$\begin{array}{rrrc}\mathrm{Cl} & 2.38496577 & 0.00030450 & 0.00000000 \\ \mathrm{Cl} & -2.38496577 & 0.00030450 & 0.00000000 \\ \mathrm{C} & 0.00000000 & 0.00011239 & 0.00000000 \\ \mathrm{H} & 0.00000000 & -0.53584403 & -0.92606298 \\ \mathrm{H} & 0.00000000 & -0.53584403 & 0.92606298 \\ \mathrm{H} & 0.00000000 & 1.07002281 & 0.00000000\end{array}$




\begin{tabular}{cccc}
\multicolumn{4}{c}{ maug-cc-pVTZ } \\
\multicolumn{4}{c}{-958.80968091705677} \\
$\mathrm{Cl}$ & 2.37559190 & 0.00120809 & 0.00000000 \\
$\mathrm{Cl}$ & -2.37559190 & 0.00120809 & 0.00000000 \\
$\mathrm{C}$ & 0.00000000 & 0.00113897 & 0.00000000 \\
$\mathrm{H}$ & 0.00000000 & -0.52818508 & -0.91755096 \\
$\mathrm{H}$ & 0.00000000 & -0.52818508 & 0.91755096 \\
$\mathrm{H}$ & 0.00000000 & 1.06043369 & 0.00000000
\end{tabular}

\section{CASPT2}

maug-cc-pVDZ

$-959.10432309123871$

$\begin{array}{cccc}\mathrm{Cl} & 2.31376762 & -0.00151493 & 0.00000000 \\ \mathrm{Cl} & -2.31376762 & -0.00151493 & 0.00000000 \\ \mathrm{C} & 0.00000000 & -0.00164893 & 0.00000000 \\ \mathrm{H} & 0.00000000 & -0.54365092 & -0.93767944 \\ \mathrm{H} & 0.00000000 & -0.54365092 & 0.93767944 \\ \mathrm{H} & 0.00000000 & 1.08141045 & 0.00000000 \\ \text { maug-cc-pVTZ } & & \\ -959.28951451935291 & \\ \mathrm{Cl} & 2.28462348 & 0.00369078 & 0.00000000 \\ \mathrm{Cl} & -2.28462348 & 0.00369078 & 0.00000000 \\ \mathrm{C} & 0.00000000 & -0.00110915 & 0.00000000 \\ \mathrm{H} & 0.00000000 & -0.53500406 & -0.92517736 \\ \mathrm{H} & 0.00000000 & -0.53500406 & 0.92517736 \\ \mathrm{H} & 0.00000000 & 1.06701810 & 0.00000000\end{array}$

\section{tPBE}

maug-cc-pVDZ

$-959.90353822437089$

$\begin{array}{cccc}\mathrm{Cl} & 2.32692323 & -0.00237364 & 0.00000000 \\ \mathrm{Cl} & -2.32692323 & -0.00237364 & 0.00000000 \\ \mathrm{C} & 0.00000000 & -0.00251253 & 0.00000000 \\ \mathrm{H} & 0.00000000 & -0.54814682 & -0.94381067 \\ \mathrm{H} & 0.00000000 & -0.54814682 & 0.94381067 \\ \mathrm{H} & 0.00000000 & 1.08762866 & 0.00000000 \\ \text { maug-cc-pVTZ } & & \\ -959.93238677916838 & \\ \mathrm{Cl} & 2.30874772 & -0.02022907 & 0.00000000 \\ \mathrm{Cl} & -2.30874772 & -0.02022907 & 0.00000000 \\ \mathrm{C} & 0.00000000 & 0.00476888 & 0.00000000 \\ \mathrm{H} & 0.00000000 & -0.53265054 & -0.93359483 \\ \mathrm{H} & 0.00000000 & -0.53265054 & 0.93359483 \\ \mathrm{H} & 0.00000000 & 1.08270641 & 0.00000000\end{array}$




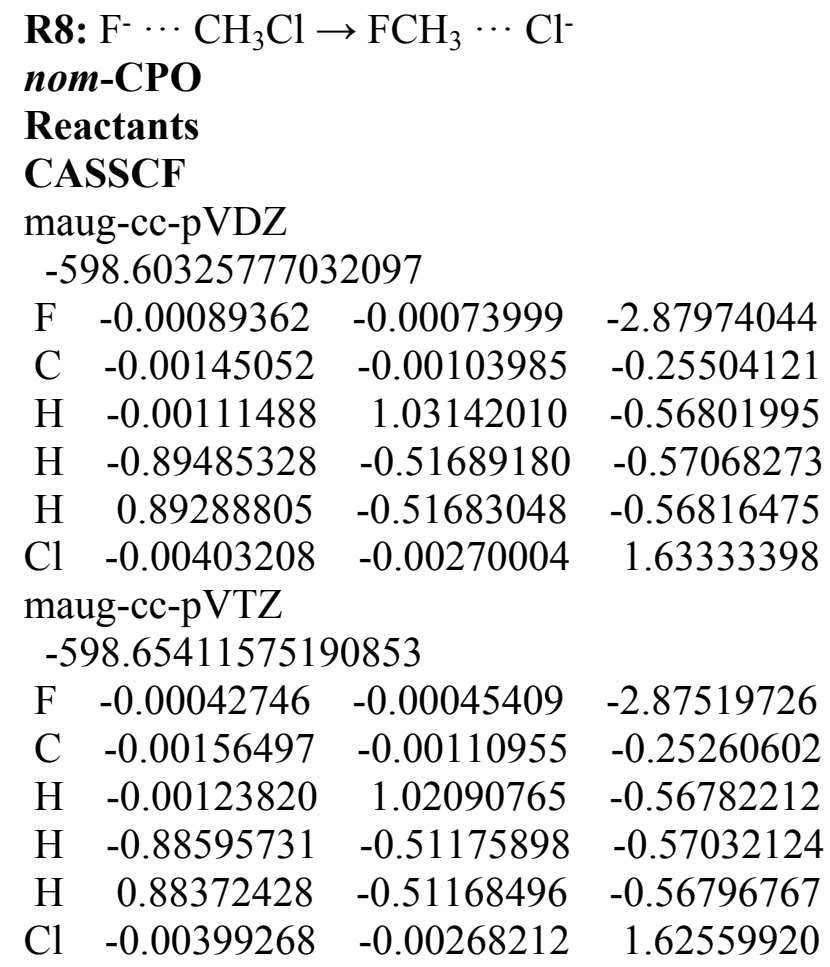

\section{CASPT2}

maug-cc-pVDZ

$-599.07321575112201$

F $\quad 0.00065079 \quad 0.00006618 \quad-2.84854641$

C $\quad-0.00194722 \quad-0.00131905 \quad-0.23923818$

$\mathrm{H} \quad-0.00189432 \quad 1.03672189 \quad-0.57674135$

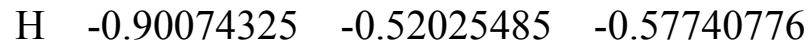

H $\quad 0.89704749 \quad-0.52011822 \quad-0.57693918$

$\begin{array}{llll}\text { Cl } & -0.00256983 & -0.00187800 & 1.61055777\end{array}$

maug-cc-pVTZ

$-599.27667952937179$

$\begin{array}{cccc}\text { F } & 0.00056777 & 0.00017867 & -2.78654026 \\ \text { C } & -0.00192696 & -0.00135085 & -0.25378228 \\ \text { H } & -0.00188671 & 1.02281060 & -0.58576074 \\ \text { H } & -0.88875998 & -0.51340988 & -0.58619603 \\ \text { H } & 0.88507778 & -0.51326515 & -0.58580929 \\ \text { Cl } & -0.00252824 & -0.00174545 & 1.58977351\end{array}$




\section{tPBE}

maug-cc-pVDZ $-599.63459456864757$

$\begin{array}{cccc}\text { F } & -0.00254102 & -0.00183153 & -2.83347930 \\ \text { C } & -0.00108332 & -0.00080578 & -0.24116145 \\ \text { H } & -0.00054336 & 1.04271790 & -0.58133383 \\ \text { H } & -0.90359051 & -0.52189590 & -0.58550556 \\ \text { H } & 0.90291311 & -0.52189407 & -0.58162341 \\ \text { Cl } & -0.00461124 & -0.00307268 & 1.61478845\end{array}$

maug-cc-pVTZ

$-599.68364247766306$

$\begin{array}{cccc}\text { F } & 0.00234380 & 0.00128208 & -2.80694216 \\ \text { C } & -0.00241096 & -0.00164152 & -0.24722048 \\ \text { H } & -0.00262596 & 1.03014504 & -0.58604160 \\ \text { H } & -0.89637248 & -0.51784435 & -0.58457745 \\ \text { H } & 0.89110605 & -0.51761464 & -0.58602040 \\ \text { Cl } & -0.00149678 & -0.00110867 & 1.60248698\end{array}$

Products

CASSCF

maug-cc-pVDZ

$-598.66128731189610$

$\begin{array}{cccc}\mathrm{F} & 0.00166296 & -0.00148667 & -2.51802641 \\ \mathrm{C} & 0.00029915 & -0.00009602 & -1.08955575 \\ \mathrm{H} & 0.00060360 & 1.03021988 & -0.75132676 \\ \mathrm{H} & -0.89231008 & -0.51425224 & -0.75066928 \\ \mathrm{H} & 0.89166362 & -0.51527208 & -0.74896649 \\ \mathrm{Cl} & -0.00065508 & 0.00227082 & 2.36050618 \\ \text { maug-cc-pVTZ } & & \\ -598.71475989858777 & \\ \mathrm{~F} & -0.00094183 & -0.00063136 & -2.50907483 \\ \mathrm{C} & 0.00109445 & -0.00035687 & -1.09510905 \\ \mathrm{H} & 0.00216702 & 1.01977707 & -0.75256426 \\ \mathrm{H} & -0.88210012 & -0.50978395 & -0.75095418 \\ \mathrm{H} & 0.88475404 & -0.51088207 & -0.75367194 \\ \mathrm{Cl} & -0.00370939 & 0.00326087 & 2.36333574\end{array}$

\section{CASPT2}

maug-cc-pVDZ

$-599.10949525910382$
F $\quad-0.00106488$
$-0.00061656$
$-2.48718760$
C $\quad 0.00090008 \quad-0.00028389-1.06284072$
$\mathrm{H} \quad 0.00216626 \quad 1.03749145 \quad-0.71079993$
$\mathrm{H} \quad-0.89773924 \quad-0.51829193 \quad-0.70918350$
$\mathrm{H} \quad 0.89963492 \quad-0.51979892 \quad-0.71168922$
$\begin{array}{llll}\mathrm{Cl} & -0.00263297 & 0.00288355 & 2.18366245\end{array}$ 
maug-cc-pVTZ

$-599.31659951262986$

$\begin{array}{rrrr}\text { F } & 0.00390104 & -0.00316676 & -2.46935589 \\ \text { C } & -0.00043593 & 0.00043738 & -1.05851119 \\ \text { H } & -0.00089219 & 1.02406191 & -0.70783911 \\ \text { H } & -0.88752850 & -0.50952840 & -0.70660946 \\ \text { H } & 0.88366479 & -0.51045013 & -0.70052593 \\ \text { Cl } & 0.00255497 & 0.00002969 & 2.14480306\end{array}$

tPBE

maug-cc-pVDZ $-599.67510399166213$

$\begin{array}{rrrr}\text { F } & 0.00112071 & -0.00119253 & -2.50261348 \\ \text { C } & 0.00037307 & -0.00014764 & -1.08012876 \\ \text { H } & 0.00078752 & 1.04263552 & -0.71912406 \\ \text { H } & -0.90298411 & -0.52062002 & -0.71849514 \\ \text { H } & 0.90272386 & -0.52167644 & -0.71751069 \\ \text { Cl } & -0.00075688 & 0.00238480 & 2.23983361\end{array}$
maug-cc-pVTZ

$-599.72473665628263$

$\begin{array}{rrrr}\text { F } & -0.00052768 & -0.00105601 & -2.49420150 \\ \text { C } & 0.00083402 & -0.00018781 & -1.07919596 \\ \text { H } & 0.00176862 & 1.03137728 & -0.71836641 \\ \text { H } & -0.89225387 & -0.51508325 & -0.71646896 \\ \text { H } & 0.89407167 & -0.51618718 & -0.71834697 \\ \text { Cl } & -0.00262860 & 0.00252066 & 2.22854129\end{array}$

Transition state

CASSCF

maug-cc-pVDZ

$-598.59585416893776$

\begin{tabular}{cccc}
$\mathrm{F}$ & 0.00000000 & 0.00000705 & -2.58973877 \\
$\mathrm{C}$ & 0.00000000 & 0.00000016 & -0.49885966 \\
$\mathrm{H}$ & 0.00000000 & 1.06504866 & -0.60851038 \\
$\mathrm{H}$ & -0.92235860 & -0.53252286 & -0.60851883 \\
$\mathrm{H}$ & 0.92235860 & -0.53252286 & -0.60851883 \\
$\mathrm{Cl}$ & 0.00000000 & -0.00001106 & 1.68445850 \\
maug-cc-pVTZ & \\
\multicolumn{4}{c}{-598.64602119878100} \\
$\mathrm{~F}$ & 0.00000000 & 0.00000750 & -2.57622527 \\
$\mathrm{C}$ & 0.00000000 & -0.00000060 & -0.49755549 \\
$\mathrm{H}$ & 0.00000000 & 1.05418996 & -0.60960264 \\
$\mathrm{H}$ & -0.91295595 & -0.52709506 & -0.60960677 \\
$\mathrm{H}$ & 0.91295595 & -0.52709506 & -0.60960677 \\
$\mathrm{Cl}$ & 0.00000000 & -0.00000767 & 1.67290897
\end{tabular}




\section{CASPT2}

maug-cc-pVDZ

$-599.06403587718432$

$\begin{array}{crrr}\text { F } & 0.00000000 & 0.00011139 & -2.52541474 \\ \mathrm{C} & 0.00000000 & -0.00004593 & -0.52526147 \\ \mathrm{H} & 0.00000000 & 1.08003469 & -0.60852000 \\ \mathrm{H} & -0.93536323 & -0.54007460 & -0.60842160 \\ \mathrm{H} & 0.93536323 & -0.54007460 & -0.60842160 \\ \mathrm{Cl} & 0.00000000 & 0.00004810 & 1.64635144\end{array}$

maug-cc-pVTZ

$-599.26989604227640$

F $\quad 0.00000000 \quad 0.00003032 \quad-2.50085443$

C $\quad 0.00000000 \quad-0.00002263 \quad-0.50798148$

$\mathrm{H} \quad 0.00000000 \quad 1.06338556 \quad-0.61180448$

$\mathrm{H} \quad-0.92091963 \quad-0.53170019 \quad-0.61176309$

H $\quad 0.92091963 \quad-0.53170019 \quad-0.61176309$

$\begin{array}{llll}\mathrm{Cl} & 0.00000000 & 0.00000617 & 1.61447857\end{array}$

\section{tPBE}

maug-cc-pVDZ

$-599.63074367573506$

$\begin{array}{rrrr}\text { F } & 0.00000000 & -0.00001648 & -2.56159601 \\ \mathrm{C} & 0.00000000 & 0.00000615 & -0.45544249 \\ \mathrm{H} & 0.00000000 & 1.07968512 & -0.61469353 \\ \mathrm{H} & -0.93502629 & -0.53982911 & -0.61471509 \\ \mathrm{H} & 0.93502629 & -0.53982911 & -0.61471509 \\ \mathrm{Cl} & 0.00000000 & -0.00001748 & 1.63147423\end{array}$

maug-cc-pVTZ

$-599.67965675157870$

F $\quad 0.00000000 \quad-0.00001882 \quad-2.54370887$

C $\quad 0.00000000 \quad 0.00000522 \quad-0.46847915$

$\mathrm{H} \quad 0.00000000 \quad 1.06917322 \quad-0.61451351$

$\mathrm{H} \quad-0.92592703 \quad-0.53457335 \quad-0.61453396$

$\mathrm{H} \quad 0.92592703 \quad-0.53457335 \quad-0.61453396$

Cl $0.00000000 \quad-0.00001386 \quad 1.62608146$

\section{mod-CPO}

\section{Reactants}

CASSCF

maug-cc-pVDZ

$-598.72189791154358$
F 0.00002889
$-0.00011577-2.92109210$
C $\quad-0.00165508 \quad-0.00118041 \quad-0.23801043$
$\mathrm{H} \quad-0.00132463 \quad 1.02930736 \quad-0.55742145$
$\mathrm{H} \quad-0.89339621 \quad-0.51607681 \quad-0.55985440$
H $\quad 0.89097948 \quad-0.51599987 \quad-0.55752892$
$\begin{array}{llll}\mathrm{Cl} & -0.00408878 & -0.00271657 & 1.62559219\end{array}$ 
maug-cc-pVTZ

$-598.78062498181316$

$\begin{array}{cccc}\text { F } & 0.00165195 & 0.00092675 & -2.92233927 \\ \text { C } & -0.00209202 & -0.00146121 & -0.23400065 \\ \text { H } & -0.00188581 & 1.01880175 & -0.55581705 \\ \text { H } & -0.88526531 & -0.51142043 & -0.55722406 \\ \text { H } & 0.88163785 & -0.51129192 & -0.55586735 \\ \text { Cl } & -0.00350300 & -0.00233699 & 1.61693328\end{array}$

\section{CASPT2}

maug-cc-pVDZ

$-599.06819552740660$

$\begin{array}{cccc}\mathrm{Cl} & -0.00000526 & -0.00001617 & -0.01046726 \\ \mathrm{C} & -0.00001066 & -0.00002291 & 1.83634387 \\ \mathrm{~F} & 0.00043566 & -0.00003240 & 4.43712748 \\ \mathrm{H} & 1.03758674 & 0.00001395 & 2.17615330 \\ \mathrm{H} & -0.51879080 & 0.89860705 & 2.17616706 \\ \mathrm{H} & -0.51877368 & -0.89854952 & 2.17620056 \\ \text { maug-cc-pVTZ } & & \\ -599.26964074491775 & \\ \mathrm{Cl} & 0.00029478 & 0.00138639 & 0.01182551 \\ \mathrm{C} & -0.00166329 & 0.00147520 & 1.84712159 \\ \mathrm{~F} & -0.00392443 & 0.00159393 & 4.38134951 \\ \mathrm{H} & 1.02211078 & -0.00398834 & 2.17744487 \\ \mathrm{H} & -0.51031844 & 0.88812924 & 2.18681705 \\ \mathrm{H} & -0.50605740 & -0.88859642 & 2.18696647\end{array}$

\section{tPBE}

maug-cc-pVDZ

$-599.60246667461888$

$\begin{array}{cccc}\mathrm{F} & -0.00015943 & -0.00016146 & -2.84851476 \\ \mathrm{C} & -0.00172008 & -0.00124759 & -0.23838678 \\ \mathrm{H} & -0.00148758 & 1.04217889 & -0.58098747 \\ \mathrm{H} & -0.90487078 & -0.52274628 & -0.58259803 \\ \mathrm{H} & 0.90209334 & -0.52264245 & -0.58099929 \\ \mathrm{Cl} & -0.00331182 & -0.00216318 & 1.62317124 \\ \text { maug-cc-pVTZ } & & \\ -599.65043704022662 & \\ \mathrm{~F} & 0.00369564 & 0.00218343 & -2.81825866 \\ \mathrm{C} & -0.00276544 & -0.00188030 & -0.24256210 \\ \mathrm{H} & -0.00314279 & 1.02910436 & -0.58471140 \\ \mathrm{H} & -0.89642601 & -0.51791775 & -0.58189066 \\ \mathrm{H} & 0.88999694 & -0.51762199 & -0.58459050 \\ \mathrm{Cl} & -0.00081468 & -0.00064981 & 1.60369822\end{array}$




\section{Products}

\section{CASSCF}

maug-cc-pVDZ

$-598.76605776517840$

$\begin{array}{rrrr}\mathrm{F} & -0.00000523 & -0.00104004 & -2.50678593 \\ \mathrm{C} & 0.00062564 & -0.00016546 & -1.06485134 \\ \mathrm{H} & 0.00137001 & 1.03122522 & -0.73147451 \\ \mathrm{H} & -0.89254502 & -0.51503472 & -0.73011604 \\ \mathrm{H} & 0.89353484 & -0.51608969 & -0.73101127 \\ \mathrm{Cl} & -0.00171608 & 0.00248838 & 2.26620058\end{array}$

maug-cc-pVTZ

$-598.82978350113035$

$\begin{array}{rrrr}\text { F } & 0.00148415 & -0.00120324 & -2.50297551 \\ \mathrm{C} & 0.00036146 & -0.00016971 & -1.07752145 \\ \mathrm{H} & 0.00070006 & 1.02109224 & -0.74002787 \\ \mathrm{H} & -0.88443469 & -0.50992846 & -0.73963492 \\ \mathrm{H} & 0.88402183 & -0.51094669 & -0.73822792 \\ \mathrm{Cl} & -0.00086864 & 0.00253956 & 2.30034915\end{array}$

\section{CASPT2}

maug-cc-pVDZ

$-599.11637407590820$

$\begin{array}{rrrl}\mathrm{F} & -0.00406046 & 0.00552144 & -2.49134271 \\ \mathrm{C} & 0.00208423 & 0.00020168 & -1.06480354 \\ \mathrm{H} & 0.00765588 & 1.03533695 & -0.70941880 \\ \mathrm{H} & -0.90120171 & -0.52067177 & -0.71125055 \\ \mathrm{H} & 0.90468368 & -0.52812061 & -0.71997386 \\ \mathrm{Cl} & -0.00789751 & 0.00911611 & 2.1987510\end{array}$

maug-cc-pVTZ

$-599.31395659742395$

$\begin{array}{cccc}\mathrm{F} & -0.00000588 & -0.00003402 & 0.01825151 \\ \mathrm{C} & -0.00005227 & -0.00011070 & 1.42743504 \\ \mathrm{Cl} & 0.00040385 & -0.00028603 & 4.64847606 \\ \mathrm{H} & 1.02244254 & 0.00020514 & 1.78300449 \\ \mathrm{H} & -0.51105540 & 0.88549113 & 1.78301359 \\ \mathrm{H} & -0.51116184 & -0.88526552 & 1.78382730\end{array}$

\section{tPBE}

maug-cc-pVDZ

$-599.66398961978598$
F $\quad 0.00140512 \quad-0.00092787 \quad-2.55639237$
C $\quad 0.00097392 \quad-0.00088353 \quad-1.13470821$
$\begin{array}{llll}\mathrm{H} & 0.00590991 & 1.04379651 & -0.77756517\end{array}$

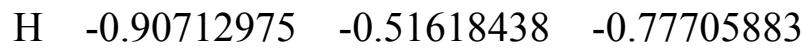
H $\quad 0.90385446 \quad-0.52490998 \quad-0.77657227$
$\begin{array}{llll}\mathrm{Cl} & -0.00374955 & 0.00049306 & 2.52425846\end{array}$ 


\begin{tabular}{|c|c|c|c|}
\hline \multicolumn{4}{|c|}{ maug-cc-pVTZ } \\
\hline $\mathrm{F}$ & -0.00013022 & -0.00084298 & -2.502 \\
\hline $\mathrm{C}$ & 0.00073773 & -0.00025120 & -1.08 \\
\hline $\mathrm{H}$ & 0.00155107 & 1.03152285 & -0.72085222 \\
\hline $\mathrm{H}$ & -0.89274283 & -0.51538021 & -0.7196278 \\
\hline $\mathrm{H}$ & 0.89411537 & -0.51646847 & -0.720880 \\
\hline & -0.00226696 & 0.00280369 & 2.247437 \\
\hline
\end{tabular}

\section{Transition state}

\section{CASSCF}

maug-cc-pVDZ

$-598.71370538492704$

$\begin{array}{cccc}\mathrm{F} & 0.00000000 & 0.00001201 & -2.60050600 \\ \mathrm{C} & 0.00000000 & 0.00000014 & -0.49968833 \\ \mathrm{H} & 0.00000000 & 1.06502735 & -0.60466288 \\ \mathrm{H} & -0.92233986 & -0.53251185 & -0.60467539 \\ \mathrm{H} & 0.92233986 & -0.53251185 & -0.60467539 \\ \mathrm{Cl} & 0.00000000 & -0.00001664 & 1.68452001\end{array}$

maug-cc-pVTZ

$-598.77217182027482$

$\begin{array}{rrrr}\text { F } & 0.00000000 & 0.00025636 & -2.59133540 \\ \text { C } & 0.00000000 & -0.00023751 & -0.49528161 \\ \text { H } & 0.00000000 & 1.05369929 & -0.60448669 \\ \text { H } & -0.91267688 & -0.52721662 & -0.60558716 \\ \text { H } & 0.91267688 & -0.52721662 & -0.60558716 \\ \text { Cl } & 0.00000000 & 0.00071425 & 1.67259004\end{array}$

\section{CASPT2}

maug-cc-pVDZ

$-599.05939269411419$

$\begin{array}{cccc}\mathrm{F} & 0.00000000 & 0.00447940 & -2.52664142 \\ \mathrm{C} & 0.00000000 & -0.00175297 & -0.50892473 \\ \mathrm{H} & 0.00000000 & 1.07714119 & -0.61249537 \\ \mathrm{H} & -0.93467219 & -0.54102984 & -0.60889461 \\ \mathrm{H} & 0.93467219 & -0.54102984 & -0.60889461 \\ \mathrm{Cl} & 0.00000000 & 0.00219121 & 1.63616276 \\ \text { maug-cc-pVTZ } & & \\ -599.26291711243596 & \\ \mathrm{~F} & 0.00000000 & 0.00000504 & -2.49931025 \\ \mathrm{C} & 0.00000000 & -0.00001085 & -0.50305395 \\ \mathrm{H} & 0.00000000 & 1.06313501 & -0.61205880 \\ \mathrm{H} & -0.92068572 & -0.53154869 & -0.61206350 \\ \mathrm{H} & 0.92068572 & -0.53154869 & -0.61206350 \\ \mathrm{Cl} & 0.00000000 & -0.00003267 & 1.60886202\end{array}$




\section{tPBE}

maug-cc-pVDZ $-599.59713728206941$

$\begin{array}{cccc}\text { F } & 0.00000000 & 0.00000138 & -2.54235697 \\ \mathrm{C} & 0.00000000 & 0.00000065 & -0.48303067 \\ \mathrm{H} & 0.00000000 & 1.08328516 & -0.61466567 \\ \mathrm{H} & -0.93814862 & -0.54163769 & -0.61470530 \\ \mathrm{H} & 0.93814862 & -0.54163769 & -0.61470530 \\ \mathrm{Cl} & 0.00000000 & -0.00001266 & 1.63977593 \\ \text { maug-cc-pVTZ } & & \\ -599.64253783433048 & \\ \mathrm{~F} & 0.00000000 & -0.00000513 & -2.52103767 \\ \mathrm{C} & 0.00000000 & 0.00000330 & -0.49792398 \\ \mathrm{H} & 0.00000000 & 1.07264387 & -0.61387519 \\ \mathrm{H} & -0.92893378 & -0.53630776 & -0.61391404 \\ \mathrm{H} & 0.92893378 & -0.53630776 & -0.61391404 \\ \mathrm{Cl} & 0.00000000 & -0.00002737 & 1.63097693\end{array}$

R9: $\mathrm{OH}^{-}+\mathrm{CH}_{3} \mathrm{~F} \rightarrow \mathrm{HOCH}_{3}+\mathrm{F}^{-}$

nom-CPO

\section{Reactants}

\section{CASSCF}

maug-cc-pVDZ

$-214.48801622$

$\begin{array}{cccc}\text { F } & 0.05021025 & -0.00800240 & 0.02164727 \\ \mathrm{C} & -0.01477505 & 0.00310330 & 1.42263036 \\ \mathrm{H} & 1.00180387 & 0.00372735 & 1.81072628 \\ \mathrm{H} & -0.54461339 & 0.90181920 & 1.73187690 \\ \mathrm{H} & -0.54885568 & -0.88814746 & 1.74586919 \\ \mathrm{O} & -40.21398752 & 0.20864420 & 0.42788806 \\ \mathrm{H} & -40.95171248 & 0.21545580 & 1.02701194 \\ \text { maug-cc-pVTZ } & & \\ -214.55053881 & & \\ \mathrm{~F} & 0.04979891 & -0.00793216 & 0.03050367 \\ \mathrm{C} & -0.01460450 & 0.00307392 & 1.41895659 \\ \mathrm{H} & 0.99269070 & 0.00373224 & 1.80857253 \\ \mathrm{H} & -0.53995526 & 0.89382592 & 1.73042476 \\ \mathrm{H} & -0.54415985 & -0.88019992 & 1.74429245 \\ \mathrm{O} & -40.21652501 & 0.20866763 & 0.42994882 \\ \mathrm{H} & -40.94917499 & 0.21543237 & 1.02495118\end{array}$




\section{CASPT2}

maug-cc-pVDZ

$-215.00145938$

$\begin{array}{llcc}\text { F } & 0.05025537 & -0.00801210 & 0.02070428 \\ \text { C } & -0.01434916 & 0.00302041 & 1.41362440 \\ \text { H } & 1.01009181 & 0.00374025 & 1.81443128 \\ \text { H } & -0.54897655 & 0.90918534 & 1.73494580 \\ \text { H } & -0.55325146 & -0.89543391 & 1.74904424 \\ \text { O } & -40.20514182 & 0.20856253 & 0.42070426 \\ \text { H } & -40.96055818 & 0.21553747 & 1.03419574\end{array}$

maug-cc-pVTZ

$-215.21496952$

$\begin{array}{cccc}\text { F } & 0.04975327 & -0.00792415 & 0.03153781 \\ \text { C } & -0.01427970 & 0.00301869 & 1.41166278 \\ \text { H } & 0.99590947 & 0.00374671 & 1.81081323 \\ \text { H } & -0.54169275 & 0.89671498 & 1.73241151 \\ \text { H } & -0.54592030 & -0.88305623 & 1.74632467 \\ \text { O } & -40.20823915 & 0.20859113 & 0.42321967 \\ \text { H } & -40.95746085 & 0.21550887 & 1.03168033\end{array}$

\section{tPBE}

maug-cc-pVDZ

$-215.29926884$

$\begin{array}{cccc}\text { F } & 0.05035901 & -0.00803642 & 0.01851674 \\ \mathrm{C} & -0.01417974 & 0.00299120 & 1.40996826 \\ \mathrm{H} & 1.01573110 & 0.00379076 & 1.81664239 \\ \mathrm{H} & -0.55188314 & 0.91411711 & 1.73673211 \\ \mathrm{H} & -0.55625723 & -0.90036265 & 1.75089050 \\ \mathrm{O} & -40.20341109 & 0.20854655 & 0.41929869 \\ \mathrm{H} & -40.96228891 & 0.21555345 & 1.03560131 \\ \text { maug-cc-pVTZ } & & \\ -215.35874527 & & \\ \mathrm{~F} & 0.05008798 & -0.00798943 & 0.02431663 \\ \mathrm{C} & -0.01416441 & 0.00300220 & 1.40924314 \\ \mathrm{H} & 1.00518614 & 0.00375504 & 1.81446402 \\ \mathrm{H} & -0.54653703 & 0.90493471 & 1.73534990 \\ \mathrm{H} & -0.55080269 & -0.89120252 & 1.74937632 \\ \mathrm{O} & -40.20625675 & 0.20857282 & 0.42160972 \\ \mathrm{H} & -40.95944325 & 0.21552718 & 1.03329028\end{array}$




\section{Products}

\section{CASSCF}

maug-cc-pVDZ

$-214.5222721$

$\begin{array}{cccc}\mathrm{C} & -0.04755853 & 0.66958827 & -0.00000000 \\ \mathrm{O} & -0.04088192 & -0.76230705 & -0.00000000 \\ \mathrm{H} & -1.09050192 & 0.97863521 & 0.00000000 \\ \mathrm{H} & 0.85679381 & -1.05900983 & 0.00000000 \\ \mathrm{H} & 0.44002428 & 1.07204670 & 0.89180086 \\ \mathrm{H} & 0.44002428 & 1.07204670 & -0.89180086 \\ \mathrm{~F} & -40.0000000 & 0.00000000 & 0.00000000 \\ \text { maug-cc-pVTZ } & & \\ -214.5799376 & & \\ \mathrm{C} & -0.03578981 & 0.51189213 & 0.00000000 \\ \mathrm{O} & -0.07414005 & -0.91678378 & 0.00000000 \\ \mathrm{H} & -1.06024360 & 0.85185160 & -0.00000000 \\ \mathrm{H} & 0.80619018 & -1.24666949 & -0.00000000 \\ \mathrm{H} & 0.46094164 & 0.89535477 & 0.88451987 \\ \mathrm{H} & 0.46094164 & 0.89535477 & -0.88451987 \\ \mathrm{~F} & -40.0000000 & 0.00000000 & 0.00000000\end{array}$

\section{CASPT2}

maug-cc-pVDZ

$\begin{array}{cccc}-215.0394301 & & \\ \mathrm{C} & -0.04680852 & 0.65975256 & -0.00000000 \\ \mathrm{O} & -0.05258911 & -0.76287892 & -0.00000000 \\ \mathrm{H} & -1.09741369 & 0.98196278 & 0.00000000 \\ \mathrm{H} & 0.86671338 & -1.05773366 & 0.00000000 \\ \mathrm{H} & 0.44399897 & 1.07494862 & 0.89893947 \\ \mathrm{H} & 0.44399897 & 1.07494862 & -0.89893947 \\ \mathrm{~F} & -40.0000000 & 0.00000000 & 0.00000000 \\ \text { maug-cc-pVTZ } & & \\ -215.2473087 & & \\ \mathrm{C} & -0.04625733 & 0.66284339 & -0.00000000 \\ \mathrm{O} & -0.04834056 & -0.75546042 & 0.00000000 \\ \mathrm{H} & -1.08440662 & 0.97783303 & 0.00000000 \\ \mathrm{H} & 0.86181540 & -1.05767646 & -0.00000000 \\ \mathrm{H} & 0.43754456 & 1.07173023 & 0.88759306 \\ \mathrm{H} & 0.43754456 & 1.07173023 & -0.88759306 \\ \mathrm{~F} & -40.0000000 & 0.00000000 & 0.00000000\end{array}$




\section{tPBE}

maug-cc-pVDZ

$-215.3363126$

$\begin{array}{cccc}\mathrm{C} & -0.04554516 & 0.65608852 & 0.00000000 \\ \mathrm{O} & -0.05209681 & -0.76072935 & -0.00000000 \\ \mathrm{H} & -1.10187712 & 0.98335863 & 0.00000000 \\ \mathrm{H} & 0.86947154 & -1.07071029 & 0.00000000 \\ \mathrm{H} & 0.44397377 & 1.08149624 & 0.90387770 \\ \mathrm{H} & 0.44397377 & 1.08149625 & -0.90387771 \\ \mathrm{~F} & -40.0000000 & 0.00000000 & 0.00000000 \\ \text { maug-cc-pVTZ } & & \\ -215.3920843 & & \\ \mathrm{C} & -0.04599095 & 0.65973819 & 0.00000000 \\ \mathrm{O} & -0.04815453 & -0.75724693 & 0.00000000 \\ \mathrm{H} & -1.09314089 & 0.98113839 & -0.00000000 \\ \mathrm{H} & 0.86568814 & -1.06998840 & -0.00000000 \\ \mathrm{H} & 0.43974912 & 1.07867937 & 0.89549963 \\ \mathrm{H} & 0.43974912 & 1.07867937 & -0.89549963 \\ \mathrm{~F} & -40.0000000 & 0.00000000 & 0.00000000\end{array}$

Transition state

CASSCF

maug-cc-pVDZ

$-214.47899995292275$

$\begin{array}{lrrr}\text { F } & 0.05035711 & -0.01019392 & -0.05165190 \\ \text { C } & -0.02312653 & 0.00586340 & 1.75968934 \\ \text { H } & 1.04335529 & 0.00500673 & 1.85421959 \\ \text { H } & -0.55323874 & 0.93642946 & 1.75965692 \\ \text { H } & -0.55617561 & -0.92287523 & 1.77593381 \\ \text { O } & -0.01830092 & 0.02351553 & 3.77854103 \\ \text { H } & -0.91297661 & 0.02716700 & 4.09437721\end{array}$

maug-cc-pVTZ

$-214.53746126819289$

F $\quad 0.04305624 \quad-0.00991019 \quad-0.03469744$

$\begin{array}{llll}\text { C } & -0.02195588 & 0.00598074 & 1.76901462\end{array}$

$\mathrm{H} \quad 1.03484928 \quad 0.00462027 \quad 1.85180510$

$\begin{array}{llll}\mathrm{H} & -0.54662526 & 0.92735007 & 1.76903520\end{array}$

$\mathrm{H} \quad-0.55024757 \quad-0.91319416 \quad 1.78504942$

$\begin{array}{llll}\text { O } & -0.01706602 & 0.02340639 & 3.76820443\end{array}$

$\mathrm{H} \quad-0.91211680 \quad 0.02665986 \quad 4.06235466$ 


\section{CASPT2}

maug-cc-pVDZ

$-215.00793658905559$

$\begin{array}{crrr}\text { F } & 0.03759865 & -0.00961903 & -0.00924967 \\ \mathrm{C} & -0.02163678 & 0.00565482 & 1.74450666 \\ \mathrm{H} & 1.05720478 & 0.00500847 & 1.86115835 \\ \mathrm{H} & -0.55784115 & 0.94867621 & 1.77661706 \\ \mathrm{H} & -0.56073172 & -0.93501905 & 1.79293806 \\ \mathrm{O} & 0.00110632 & 0.02306628 & 3.75726820 \\ \mathrm{H} & -0.92580610 & 0.02714527 & 4.04752734\end{array}$

maug-cc-pVTZ

$-215.22179768444886$

$\begin{array}{cccc}\text { F } & 0.02467855 & -0.00934494 & 0.02232481 \\ \text { C } & -0.01986554 & 0.00576924 & 1.75837610 \\ \text { H } & 1.04567592 & 0.00505599 & 1.85825717 \\ \text { H } & -0.54849914 & 0.93535831 & 1.79226015 \\ \text { H } & -0.55125960 & -0.92151643 & 1.80835950 \\ \text { O } & 0.00388428 & 0.02287873 & 3.73745608 \\ \text { H } & -0.92472048 & 0.02671207 & 3.99373219\end{array}$

tPBE

maug-cc-pVDZ $-215.30468700070128$

$\begin{array}{cccc}\text { F } & 0.03855100 & -0.00964408 & -0.00965341 \\ \text { C } & -0.02166744 & 0.00542940 & 1.71857974 \\ \text { H } & 1.06153923 & 0.00506915 & 1.86452813 \\ \text { H } & -0.56083845 & 0.95461244 & 1.77620964 \\ \text { H } & -0.56369070 & -0.94098361 & 1.79264528 \\ \text { O } & 0.00617048 & 0.02320843 & 3.77548453 \\ \text { H } & -0.93017013 & 0.02722124 & 4.05297209\end{array}$
maug-cc-pVTZ $-215.36272654325612$

$\begin{array}{cccc}\text { F } & 0.02792925 & -0.00954377 & 0.00064021 \\ \text { C } & -0.01905137 & 0.00553688 & 1.73149288 \\ \text { H } & 1.05415871 & 0.00504865 & 1.85837197 \\ \text { H } & -0.55215574 & 0.94399084 & 1.78783701 \\ \text { H } & -0.55493940 & -0.93020935 & 1.80409119 \\ \text { O } & 0.00534422 & 0.02315238 & 3.77093389 \\ \text { H } & -0.93139168 & 0.02693735 & 4.01739884\end{array}$




\section{mod-CPO}

Reactants

CASSCF

maug-cc-pVDZ

$-214.6465412$

$\begin{array}{cccc}\text { F } & 0.05043022 & -0.00803987 & 0.01687876 \\ \text { C } & -0.01487784 & 0.00312060 & 1.42485855 \\ \text { H } & 1.00237305 & 0.00373285 & 1.81160248 \\ \text { H } & -0.54495532 & 0.90235350 & 1.73270458 \\ \text { H } & -0.54920010 & -0.88866708 & 1.74670563 \\ \text { O } & -40.20317114 & 0.20854433 & 0.41910382 \\ \text { H } & -40.96252886 & 0.21555567 & 1.03579618\end{array}$

maug-cc-pVTZ

$-214.7133875$

F $\quad 0.04998654 \quad-0.00796435 \quad 0.02645710$

$\begin{array}{llll}\mathrm{C} & -0.01470117 & 0.00309064 & 1.42105177\end{array}$

$\begin{array}{llll}\mathrm{H} & 0.99327276 & 0.00373608 & 1.80925133\end{array}$

$\begin{array}{llll}\mathrm{H} & -0.54029059 & 0.89436111 & 1.73105700\end{array}$

$\begin{array}{llll}\mathrm{H} & -0.54449754 & -0.88072349 & 1.74493280\end{array}$

$\begin{array}{llll}\text { O } & -40.20580447 & 0.20856865 & 0.42124241\end{array}$

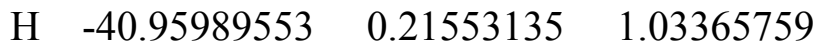

\section{CASPT2}

maug-cc-pVDZ

$-214.9979014$

$\begin{array}{cccc}\mathrm{F} & 0.05028837 & -0.00802175 & 0.02003261 \\ \mathrm{C} & -0.01434338 & 0.00302103 & 1.41332012 \\ \mathrm{H} & 1.01036164 & 0.00373764 & 1.81476951 \\ \mathrm{H} & -0.54913482 & 0.90943191 & 1.73526622 \\ \mathrm{H} & -0.55340180 & -0.89566884 & 1.74936154 \\ \mathrm{O} & -40.20448275 & 0.20846227 & 0.44248450 \\ \mathrm{H} & -40.97121725 & 0.21563773 & 1.04241550\end{array}$

maug-cc-pVTZ

$-215.2069243$

$\begin{array}{llll}\text { F } & 0.04980236 & -0.00793678 & 0.03046705\end{array}$

$\begin{array}{llll}\text { C } & -0.01421383 & 0.00300438 & 1.41076495\end{array}$

$\begin{array}{llll}\mathrm{H} & 0.99574762 & 0.00374616 & 1.81145881\end{array}$

$\begin{array}{llll}\mathrm{H} & -0.54167824 & 0.89662360 & 1.73307894\end{array}$

$\begin{array}{llll}\mathrm{O} & -0.20771174 & 0.20858626 & 0.42279135\end{array}$

$\begin{array}{llll}\mathrm{H} & -40.95798826 & 0.21551374 & 1.03210865\end{array}$

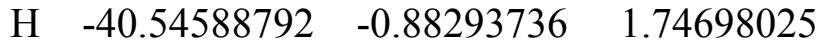




\section{tPBE}

maug-cc-pVDZ

$-215.2643031$

$\begin{array}{llcc}\text { F } & 0.05056951 & -0.00805757 & 0.01383695 \\ \text { C } & -0.01425816 & 0.00302933 & 1.41112157 \\ \text { H } & 1.01586703 & 0.00371247 & 1.81783991 \\ \text { H } & -0.55207823 & 0.91438209 & 1.73787084 \\ \text { H } & -0.55633015 & -0.90056631 & 1.75208073 \\ \text { O } & -40.20190109 & 0.20853261 & 0.41807238 \\ \text { H } & -40.96379891 & 0.21556739 & 1.03682762\end{array}$

maug-cc-pVTZ

$-215.3224605$

$\begin{array}{cccc}\text { F } & 0.05031915 & -0.00803608 & 0.01947491 \\ \text { C } & -0.01425409 & 0.00301886 & 1.41138601 \\ \text { H } & 1.00572309 & 0.00375603 & 1.81538110 \\ \text { H } & -0.54688218 & 0.90542982 & 1.73624469 \\ \text { H } & -0.55113597 & -0.89166863 & 1.75026330 \\ \text { O } & -40.20457033 & 0.20855725 & 0.42024013 \\ \text { H } & -40.96112967 & 0.21554275 & 1.03465987\end{array}$

\section{Products}

CASSCF

maug-cc-pVDZ

$\begin{array}{cccc}-214.6805562 & & \\ \mathrm{C} & -0.04808477 & 0.67396557 & -0.00000000 \\ \mathrm{O} & -0.05381317 & -0.76684658 & 0.00000000 \\ \mathrm{H} & -1.09139737 & 0.97988254 & 0.00000000 \\ \mathrm{H} & 0.86933854 & -1.06163151 & -0.00000000 \\ \mathrm{H} & 0.44092839 & 1.07281499 & 0.89243671 \\ \mathrm{H} & 0.44092839 & 1.07281499 & -0.89243671 \\ \mathrm{~F} & -40.0000000 & 0.00000000 & 0.00000000 \\ \text { maug-cc-pVTZ } & & \\ -214.7419432 & & \\ \mathrm{C} & -0.04793902 & 0.67488936 & -0.00000000 \\ \mathrm{O} & -0.05095262 & -0.76363015 & 0.00000000 \\ \mathrm{H} & -1.08327133 & 0.97786469 & 0.00000000 \\ \mathrm{H} & 0.86539160 & -1.05852495 & -0.00000000 \\ \mathrm{H} & 0.43733568 & 1.07020052 & 0.88518992 \\ \mathrm{H} & 0.43733568 & 1.07020052 & -0.88518992 \\ \mathrm{~F} & -40.0000000 & 0.00000000 & 0.00000000\end{array}$




\section{CASPT2}

maug-cc-pVDZ

$-215.0367769$

$\begin{array}{cccc}\mathrm{C} & -0.04709891 & 0.65961456 & -0.00000000 \\ \mathrm{O} & -0.05450526 & -0.76577890 & -0.00000000 \\ \mathrm{H} & -1.09762622 & 0.98330924 & 0.00000000 \\ \mathrm{H} & 0.86813238 & -1.05490716 & 0.00000000 \\ \mathrm{H} & 0.44449901 & 1.07438113 & 0.89907961 \\ \mathrm{H} & 0.44449901 & 1.07438113 & -0.89907961 \\ \mathrm{~F} & -40.0000000 & 0.00000000 & 0.00000000\end{array}$

maug-cc-pVTZ

$-215.2399532$
C -0.04655201
$\begin{array}{ll}0.66239365 & -0.00000000\end{array}$
$\begin{array}{llll}\mathrm{O} & -0.05024172 & -0.75847393 & 0.00000000\end{array}$
$\begin{array}{llll}\mathrm{H} & -1.08447383 & 0.97935002 & -0.00000000\end{array}$
$\begin{array}{llll}\mathrm{H} & 0.86308075 & -1.05405928 & -0.00000000\end{array}$
$\begin{array}{llll}\mathrm{H} & 0.43804341 & 1.07089477 & 0.88777475\end{array}$
$\begin{array}{llll}\mathrm{H} & 0.43804341 & 1.07089477 & -0.88777475\end{array}$
$\begin{array}{llll}\text { F } & -40.0000000 & 0.00000000 & 0.00000000\end{array}$

\section{tPBE}

maug-cc-pVDZ

$-215.3020187$

$\begin{array}{cccc}\mathrm{C} & -0.04592464 & 0.65871304 & 0.000000000 \\ \mathrm{O} & -0.05233535 & -0.76473184 & 0.00000000 \\ \mathrm{H} & -1.10249567 & 0.98459242 & -0.00000000 \\ \mathrm{H} & 0.87098323 & -1.07394756 & -0.00000000 \\ \mathrm{H} & 0.44383622 & 1.08318697 & 0.90419775 \\ \mathrm{H} & 0.44383622 & 1.08318697 & -0.90419775 \\ \mathrm{~F} & -40.0000000 & 0.00000000 & 0.00000000 \\ \text { maug-cc-pVTZ } & & \\ -215.3566765 & & \\ \mathrm{C} & -0.04651372 & 0.66260589 & 0.00000000 \\ \mathrm{O} & -0.04838798 & -0.76118041 & 0.00000000 \\ \mathrm{H} & -1.09392052 & 0.98243135 & -0.00000000 \\ \mathrm{H} & 0.86755312 & -1.07426666 & -0.00000000 \\ \mathrm{H} & 0.43958455 & 1.08070492 & 0.89572715 \\ \mathrm{H} & 0.43958455 & 1.08070492 & -0.89572715 \\ \mathrm{~F} & -40.0000000 & 0.00000000 & 0.00000000\end{array}$




\section{Transition state}

\section{CASSCF}

maug-cc-pVDZ

$-214.64257050779125$

$\begin{array}{lrrr}\text { F } & 0.05585598 & -0.01029114 & -0.07941971 \\ \text { C } & -0.02374689 & 0.00571168 & 1.75021767 \\ \text { H } & 1.04169433 & 0.00500879 & 1.85292955 \\ \text { H } & -0.55342812 & 0.93562192 & 1.75350428 \\ \text { H } & -0.55621201 & -0.92240964 & 1.76963772 \\ \text { O } & -0.00587556 & 0.02349294 & 3.80289651 \\ \text { H } & -0.92839375 & 0.02777842 & 4.12099998\end{array}$

maug-cc-pVTZ

$-214.67332412899449$

F $\quad 0.03456621 \quad-0.00999275 \quad-0.04944922$

$\begin{array}{llll}\text { C } & -0.02612410 & 0.00598340 & 1.78130944\end{array}$

$\begin{array}{llll}\mathrm{H} & 1.05172507 & 0.00497445 & 1.85103038\end{array}$

$\begin{array}{llll}\mathrm{H} & -0.54803153 & 0.92834446 & 1.77098792\end{array}$

$\begin{array}{llll}\mathrm{H} & -0.55079671 & -0.91484831 & 1.78698999\end{array}$

$\begin{array}{llll}\mathrm{O} & -0.00139882 & 0.02327387 & 3.77795347\end{array}$

$\mathrm{H} \quad-0.93004614 \quad 0.02717784 \quad 4.05194402$

\section{CASPT2}

maug-cc-pVDZ

$-214.99981335943909$

$\begin{array}{crrr}\text { F } & 0.02907358 & -0.00789602 & -0.00481116 \\ \text { C } & -0.01930910 & 0.00494795 & 1.74610167 \\ \text { H } & 1.06017723 & 0.00460894 & 1.85929034 \\ \text { H } & -0.55559397 & 0.94779222 & 1.78191445 \\ \text { H } & -0.55835638 & -0.93568657 & 1.79913711 \\ \text { O } & 0.00505425 & 0.02361324 & 3.76187611 \\ \text { H } & -0.93115163 & 0.02753320 & 4.02725749\end{array}$

maug-cc-pVTZ

$-215.21114672510154$

F $\quad 0.01913689 \quad-0.00860342 \quad 0.02636704$

$\begin{array}{llll}\text { C } & -0.01974937 & 0.00550314 & 1.76376508\end{array}$

$\begin{array}{llll}\mathrm{H} & 1.05018238 & 0.00483728 & 1.85677163\end{array}$

$\mathrm{H} \quad-0.54874937 \quad 0.93514463 \quad 1.79628895$

H $\quad-0.55056759 \quad-0.92210273 \quad 1.81198657$

$\begin{array}{llll}\mathrm{O} & 0.00759425 & 0.02232907 & 3.73846443\end{array}$

$\mathrm{H} \quad-0.92795319 \quad 0.02780499 \quad 3.97712230$ 


\section{tPBE}

maug-cc-pVDZ

$-215.26869386394995$

$\begin{array}{cccc}\text { F } & 0.02962955 & -0.00976809 & -0.01092207 \\ \mathrm{C} & -0.01919615 & 0.00548995 & 1.72724242 \\ \mathrm{H} & 1.06556887 & 0.00528192 & 1.86009635 \\ \mathrm{H} & -0.55757133 & 0.95500040 & 1.78297475 \\ \mathrm{H} & -0.55997139 & -0.94153312 & 1.79956594 \\ \mathrm{O} & 0.00903050 & 0.02337049 & 3.77830927 \\ \mathrm{H} & -0.93759606 & 0.02707141 & 4.03349935\end{array}$

maug-cc-pVTZ

$-215.33914694317139$

$\begin{array}{cccc}\text { F } & 0.02294018 & -0.00964109 & -0.00518868 \\ \text { C } & -0.01608771 & 0.00558278 & 1.73419047 \\ \text { H } & 1.05693481 & 0.00497782 & 1.85457379 \\ \text { H } & -0.54992277 & 0.94358590 & 1.78891969 \\ \text { H } & -0.55282437 & -0.92966820 & 1.80524323 \\ \text { O } & 0.00632979 & 0.02338091 & 3.77835799 \\ \text { H } & -0.93747596 & 0.02669484 & 4.01466952\end{array}$

R10: $\mathrm{H}+\mathrm{N}_{2} \rightarrow \mathrm{HN}_{2}$

nom-CPO

Reactants

CASSCF

maug-cc-pVDZ

$-109.49028424426659$

$\begin{array}{llll}\mathrm{N} & -0.06530814 & 0.61421642 & 0.00000000\end{array}$

$\begin{array}{llll}\mathrm{N} & -0.05973552 & -0.47341035 & 0.00000000\end{array}$

H $\quad 50.87348865 \quad-0.98524307 \quad 0.00000000$

maug-cc-pVTZ

$-109.51715026623231$

$\begin{array}{llll}\mathrm{N} & -0.06757991 & 0.60913754 & 0.00000000\end{array}$

$\begin{array}{llll}\mathrm{N} & -0.05749917 & -0.46828431 & 0.00000000\end{array}$

$\begin{array}{llll}\mathrm{H} & 50.87352408 & -0.98529023 & 0.00000000\end{array}$

\section{CASPT2}

maug-cc-pVDZ

$-109.75882963507256$

$\begin{array}{llll}\mathrm{N} & -0.06303854 & 0.63016007 & 0.00000000\end{array}$

$\mathrm{N} \quad-0.06189050 \quad-0.48940549 \quad 0.00000000$

H $\quad 50.87337404 \quad-0.98519158 \quad 0.00000000$

maug-cc-pVTZ

$-109.84811575602085$

$\begin{array}{llll}\mathrm{N} & -0.06330916 & 0.62263930 & 0.00000000\end{array}$

$\mathrm{N} \quad-0.06167510 \quad-0.48187809 \quad 0.00000000$

H $\quad 50.87342926 \quad-0.98519821 \quad 0.00000000$ 


\section{tPBE}

maug-cc-pVDZ

$-109.90326079370763$

$\begin{array}{cccc}\mathrm{N} & -0.07333024 & 0.62506116 & 0.000000000 \\ \mathrm{~N} & -0.05163369 & -0.48408240 & 0.00000000 \\ \mathrm{H} & 50.87340893 & -0.98541576 & 0.00000000\end{array}$

maug-cc-pVTZ

$-109.92712604974987$

$\begin{array}{llll}\mathrm{N} & -0.06837449 & 0.61919510 & 0.00000000\end{array}$

$\begin{array}{llll}\mathrm{N} & -0.05663287 & -0.47832427 & 0.00000000\end{array}$

H $\quad 50.87345236 \quad-0.98530783 \quad 0.00000000$

Products

CASSCF

maug-cc-pVDZ

$-109.44636177731962$

$\begin{array}{llll}\mathrm{N} & -0.04748714 & 0.64062645 & 0.00000000\end{array}$

$\begin{array}{llll}\mathrm{N} & -0.06835161 & -0.52298430 & 0.00000000\end{array}$

$\mathrm{H} \quad 0.86428375 \quad-0.96207915 \quad 0.00000000$

maug-cc-pVTZ

$-109.47254460612912$

$\begin{array}{llll}\mathrm{N} & -0.04839425 & 0.63628920 & 0.00000000\end{array}$

$\begin{array}{llll}\mathrm{N} & -0.06332869 & -0.51956415 & 0.00000000\end{array}$

$\mathrm{H} \quad 0.86016793 \quad-0.96116204 \quad 0.00000000$

\section{CASPT2}

maug-cc-pVDZ

$-109.74961171598348$

$\begin{array}{lll}\mathrm{N} & -0.07565063 & 0.67490716\end{array}$

0.00000000

$\begin{array}{llll}\mathrm{N} & -0.05782097 & -0.51306255 & 0.00000000\end{array}$

$\mathrm{H} \quad 0.88191660 \quad-1.00628160 \quad 0.00000000$

maug-cc-pVTZ

$-109.84363028859005$

$\begin{array}{lll}\mathrm{N} & -0.07654532 & 0.66685674\end{array}$

0.00000000

$\begin{array}{llll}\mathrm{N} & -0.05021555 & -0.50727470 & 0.00000000\end{array}$

$\mathrm{H} \quad 0.87520586 \quad-1.00401904 \quad 0.00000000$

\section{tPBE}

maug-cc-pVDZ

$-109.90616488251531$

$\begin{array}{rrrr}\mathrm{N} & -0.08457191 & 0.67312906 & 0.00000000 \\ \mathrm{~N} & -0.05538710 & -0.49701858 & 0.00000000 \\ \mathrm{H} & 0.88840402 & -1.02054749 & 0.00000000\end{array}$

maug-cc-pVTZ

$-109.92944299454818$

$\begin{array}{llll}\mathrm{N} & -0.08453838 & 0.66664266 & 0.00000000\end{array}$

$\begin{array}{llll}\mathrm{N} & -0.05073161 & -0.49295590 & 0.00000000\end{array}$

$\mathrm{H} \quad 0.88371499 \quad-1.01812376 \quad 0.00000000$ 


\section{Transition state}

\section{CASSCF}

maug-cc-pVDZ

$-109.44400424375692$

$\mathrm{N} \quad 0.03939636 \quad-0.61094837$

0.00000000

$\begin{array}{lll}\mathrm{N} & 0.07974423 & 0.50921009\end{array}$

0.00000000

$\mathrm{H} \quad-1.13927159 \quad 1.08618428$

0.00000000

maug-cc-pVTZ

$-109.47013313392824$

$\begin{array}{rrrr}\mathrm{N} & 0.03802059 & -0.60387163 & 0.00000000 \\ \mathrm{~N} & 0.07288749 & 0.50757235 & 0.00000000 \\ \mathrm{H} & -1.13103909 & 1.08074528 & 0.00000000\end{array}$

\section{CASPT2}

maug-cc-pVDZ

$-109.73145362691622$

$\begin{array}{cccc}\mathrm{N} & 0.08094517 & -0.65435192 & 0.00000000 \\ \mathrm{~N} & 0.08942932 & 0.48937918 & 0.00000000 \\ \mathrm{H} & -1.19050550 & 1.14941873 & 0.00000000 \\ \text { maug-cc-pVTZ } & & \\ -109.82334459424165 & \\ \mathrm{~N} & 0.07963663 & -0.64610566 & 0.00000000 \\ \mathrm{~N} & 0.09151645 & 0.48199251 & 0.00000000 \\ \mathrm{H} & -1.19128408 & 1.14855915 & 0.00000000\end{array}$

tPBE

maug-cc-pVDZ

$-109.88189605735531$

$\begin{array}{rrrr}\mathrm{N} & 0.09953478 & -0.66494880 & 0.00000000 \\ \mathrm{~N} & 0.11215683 & 0.46174765 & 0.00000000 \\ \mathrm{H} & -1.23182261 & 1.18764714 & 0.00000000\end{array}$

maug-cc-pVTZ

$-109.90522025544129$

$\begin{array}{llll}\mathrm{N} & 0.09649865 & -0.65574202 & 0.00000000\end{array}$

$\begin{array}{llll}\mathrm{N} & 0.10702957 & 0.45951599 & 0.00000000\end{array}$

$\mathrm{H} \quad-1.22365922 \quad 1.18067203 \quad 0.00000000$

\section{mod-CPO}

\section{Reactants}

\section{CASSCF}

maug-cc-pVDZ

$-109.59492669280904$

$\begin{array}{llll}\mathrm{N} & -0.06183322 & 0.62750379 & 0.00000000\end{array}$

$\mathrm{N} \quad-0.06311550 \quad-0.48677520 \quad 0.00000000$

$\mathrm{H} \quad 50.87339372 \quad-0.98516559 \quad 0.00000000$ 
maug-cc-pVTZ

$-109.62018047661655$

$\begin{array}{cccc}\mathrm{N} & -0.06091019 & 0.62222581 & 0.00000000 \\ \mathrm{~N} & -0.06407701 & -0.48151668 & 0.00000000 \\ \mathrm{H} & 50.87343220 & -0.98514614 & 0.00000000\end{array}$

\section{CASPT2}

maug-cc-pVDZ

$-109.76127937293312$

$\mathrm{N} \quad-0.06317733 \quad 0.62953468$

N $\quad-0.06175763 \quad-0.48877698 \quad 0.00000000$

H $\quad 50.87337996 \quad-0.98519470 \quad 0.00000000$

maug-cc-pVTZ

$-109.84972623855219$

$\mathrm{N} \quad-0.06332276 \quad 0.62216858$

0.00000000

$\begin{array}{llll}\mathrm{N} & -0.06166533 & -0.48140702 & 0.00000000\end{array}$

H $\quad 50.87343309 \quad-0.98519856 \quad 0.00000000$

\section{tPBE}

maug-cc-pVDZ

$-109.88816910285784$

$\begin{array}{llll}\mathrm{N} & -0.05898968 & 0.62778486 & 0.00000000\end{array}$

$\mathrm{N} \quad-0.06595765 \quad-0.48711966 \quad 0.00000000$

H $\quad 50.87339232 \quad-0.98510220 \quad 0.00000000$

maug-cc-pVTZ

$-109.91224940415682$

$\begin{array}{llll}\mathrm{N} & -0.05115723 & 0.62163036 & 0.00000000\end{array}$

$\mathrm{N} \quad-0.07383644 \quad-0.48113350 \quad 0.00000000$

H $\quad 50.87343868 \quad-0.98493386 \quad 0.00000000$

\section{Products}

CASSCF

maug-cc-pVDZ

$-109.54712158248248$

$\begin{array}{llll}\mathrm{N} & -0.05030176 & 0.66397493 & 0.00000000\end{array}$

$\mathrm{N} \quad-0.09427130 \quad-0.52853622 \quad 0.00000000$

H $\quad 0.89301806 \quad-0.97987571 \quad 0.00000000$

maug-cc-pVTZ

$-109.57129335982468$

$\begin{array}{llll}\mathrm{N} & -0.05208619 & 0.65957043 & 0.00000000\end{array}$

$\mathrm{N} \quad-0.08869992 \quad-0.52374708 \quad 0.00000000$

$\mathrm{H} \quad 0.88923111 \quad-0.98026035 \quad 0.00000000$ 


\section{CASPT2}

maug-cc-pVDZ

$-109.74735904756248$

$\begin{array}{llll}\mathrm{N} & -0.06278433 & 0.66998853 & 0.00000000\end{array}$

$\mathrm{N} \quad-0.07082278 \quad-0.52429783 \quad 0.00000000$

H $\quad 0.88205211 \quad-0.99012769 \quad 0.00000000$

maug-cc-pVTZ

$-109.83983320386922$

$\begin{array}{llll}\mathrm{N} & -0.06615166 & 0.66282283 & 0.00000000\end{array}$

$\mathrm{N} \quad-0.05770483 \quad-0.51774722 \quad 0.00000000$

$\mathrm{H} \quad 0.87230149 \quad-0.98951261 \quad 0.00000000$

\section{tPBE}

maug-cc-pVDZ

$-109.89060582708296$

$\begin{array}{rrrr}\mathrm{N} & -0.07373866 & 0.67219597 & 0.00000000 \\ \mathrm{~N} & -0.05953723 & -0.51286715 & 0.00000000 \\ \mathrm{H} & 0.88172089 & -1.00376582 & 0.00000000\end{array}$

maug-cc-pVTZ

$-109.91416150331597$

$\begin{array}{llll}\mathrm{N} & -0.07629897 & 0.66695755 & 0.00000000\end{array}$

$\mathrm{N} \quad-0.05023968 \quad-0.50781802 \quad 0.00000000$

$\mathrm{H} \quad 0.87498365 \quad-1.00357653 \quad 0.00000000$

Transition state

CASSCF

maug-cc-pVDZ

$-109.54323238621879$

$\begin{array}{llll}\mathrm{N} & 0.03906013 & -0.62219013 & 0.00000000\end{array}$

$\begin{array}{llll}\mathrm{N} & 0.05241484 & 0.53503897 & 0.00000000\end{array}$

$\mathrm{H} \quad-1.11160597 \quad 1.07159717 \quad 0.00000000$

maug-cc-pVTZ

$-109.56786997464013$

$\begin{array}{rrrr}\mathrm{N} & 0.03827963 & -0.61536481 & 0.00000000 \\ \mathrm{~N} & 0.04529380 & 0.53286877 & 0.00000000 \\ \mathrm{H} & -1.10370443 & 1.06694204 & 0.00000000\end{array}$

\section{CASPT2}

maug-cc-pVDZ

$-109.73101640303896$

$\begin{array}{rrrr}\mathrm{N} & 0.07353891 & -0.64848368 & 0.00000000 \\ \mathrm{~N} & 0.08554100 & 0.49587552 & 0.00000000 \\ \mathrm{H} & -1.17921091 & 1.13705416 & 0.00000000\end{array}$

maug-cc-pVTZ

$-109.82181542039594$

$\begin{array}{rrrr}\mathrm{N} & 0.07277003 & -0.63981086 & 0.00000000 \\ \mathrm{~N} & 0.08442334 & 0.48898847 & 0.00000000 \\ \mathrm{H} & -1.17732438 & 1.13526839 & 0.00000000\end{array}$




\section{TPBE}

maug-cc-pVDZ

$-109.86574501452725$

$\begin{array}{cccc}\mathrm{N} & 0.09780428 & -0.66548748 & 0.00000000 \\ \mathrm{~N} & 0.10665521 & 0.46782626 & 0.00000000 \\ \mathrm{H} & -1.22459050 & 1.18210722 & 0.00000000 \\ \text { maug-cc-pVTZ } & & \\ -109.88937087328898 & \\ \mathrm{~N} & 0.09494860 & -0.65640038 & 0.00000000 \\ \mathrm{~N} & 0.10181506 & 0.46533359 & 0.00000000 \\ \mathrm{H} & -1.21689466 & 1.17551279 & 0.00000000\end{array}$

R11: $\mathrm{H}+\mathrm{C}_{2} \mathrm{H}_{4} \rightarrow \mathrm{CH}_{3} \mathrm{CH}_{2}$

nom-CPO

Reactants

CASSCF

maug-cc-pVDZ

$-78.568530943458597$

$\begin{array}{llll}\text { C } & -0.55455986 & 0.00011622 & -0.25625524\end{array}$

$\begin{array}{llll}\text { C } & 0.75789871 & 0.00001164 & 0.02670027\end{array}$

$\mathrm{H} \quad-31.52628082 \quad-0.00044957 \quad 1.57406331$

$\mathrm{H} \quad-1.10912591 \quad 0.92319506 \quad-0.37566905$

$\mathrm{H} \quad-1.10914439 \quad-0.92287344 \quad-0.37626165$

$\mathrm{H} \quad 1.31248960 \quad-0.92305720 \quad 0.14597405$

H $\quad 1.31250866 \quad 0.92299189 \quad 0.14656650$

maug-cc-pVTZ

$-78.591526262032446$

$\begin{array}{llll}\text { C } & -0.54824929 & 0.00011650 & -0.26553467\end{array}$

$\begin{array}{llll}\text { C } & 0.75154131 & 0.00001148 & 0.03481207\end{array}$

$\mathrm{H} \quad-31.52610477 \quad-0.00044932 \quad 1.57732854$

$\mathrm{H} \quad-1.09669890 \quad 0.91484348 \quad-0.39192871$

$\mathrm{H} \quad-1.09670968 \quad-0.91452192 \quad-0.39252167$

H $1.29999828 \quad-0.91472222 \quad 0.16118482$

H $\quad 1.30000905 \quad 0.91465660 \quad 0.16177782$

\section{CASPT2}

maug-cc-pVDZ

$-78.818607191121117$

$\begin{array}{cccc}\mathrm{C} & -0.55706579 & 0.00012127 & -0.26318145 \\ \mathrm{C} & 0.76038962 & 0.00000732 & 0.03284379 \\ \mathrm{H} & -31.52615346 & -0.00045156 & 1.57595710 \\ \mathrm{H} & -1.11360206 & 0.93379217 & -0.38788599 \\ \mathrm{H} & -1.11363030 & -0.93345268 & -0.38848127 \\ \mathrm{H} & 1.31690985 & -0.93366274 & 0.15763510 \\ \mathrm{H} & 1.31693814 & 0.93358083 & 0.15823091\end{array}$


maug-cc-pVTZ

$-78.900139797200126$

$\begin{array}{cccc}\mathrm{C} & -0.54895292 & 0.00011268 & -0.26508740 \\ \mathrm{C} & 0.75227187 & 0.00001468 & 0.03437398 \\ \mathrm{H} & -31.52611504 & -0.00044755 & 1.57714317 \\ \mathrm{H} & -1.09760340 & 0.92134266 & -0.39104347 \\ \mathrm{H} & -1.09760335 & -0.92103460 & -0.39164653 \\ \mathrm{H} & 1.30089444 & -0.92121888 & 0.16038772 \\ \mathrm{H} & 1.30089440 & 0.92116560 & 0.16099073\end{array}$

tPBE

maug-cc-pVDZ

$-78.957348331259951$

$\begin{array}{llll}\mathrm{C} & -0.55364267 & 0.00009630 & -0.25474311\end{array}$

C $\quad 0.75700924 \quad 0.00002866 \quad 0.02529424$

H $\quad-31.52630463 \quad-0.00044008 \quad 1.57360437$

$\mathrm{H} \quad-1.11776302 \quad 0.93677580 \quad-0.37507692$

$\mathrm{H} \quad-1.11772470 \quad-0.93652538 \quad-0.37571011$

$\mathrm{H} \quad 1.32112524 \quad-0.93665158 \quad 0.14555805$

H $\quad 1.32108654 \quad 0.93665088 \quad 0.14619168$

maug-cc-pVTZ

$-78.980359495064548$

$\begin{array}{llll}\text { C } & -0.54644692 & 0.00011439 & -0.26429074\end{array}$

$\begin{array}{llll}\text { C } & 0.74975974 & 0.00001324 & 0.03364302\end{array}$

$\mathrm{H} \quad-31.52611830 \quad-0.00044845 \quad 1.57706915$

$\mathrm{H} \quad-1.10419044 \quad 0.92751745 \quad-0.39219808$

$\mathrm{H} \quad-1.10419642 \quad-0.92720156 \quad-0.39280204$

H $\quad 1.30748628 \quad-0.92740506 \quad 0.16154648$

H $\quad 1.30749205 \quad 0.92734459 \quad 0.16215042$

\begin{tabular}{|c|c|c|c|}
\hline \multicolumn{4}{|c|}{ Products } \\
\hline \multicolumn{4}{|c|}{ CASSCF } \\
\hline \multicolumn{4}{|c|}{ maug-cc-pVDZ } \\
\hline $\mathrm{C}$ & -0.25482825 & -0.81233843 & -0.00258914 \\
\hline $\mathrm{C}$ & -0.21580201 & 0.68375335 & -0.00558309 \\
\hline $\mathrm{H}$ & 0.77413626 & -1.24937657 & 0.06638383 \\
\hline $\mathrm{H}$ & -0.82047299 & -1.19688811 & 0.84885605 \\
\hline $\mathrm{H}$ & -0.70488110 & -1.20347109 & -0.9176105 \\
\hline $\mathrm{H}$ & 0.04200954 & 1.21350857 & -0.91458494 \\
\hline $\mathrm{H}$ & -0.07719943 & 1.22029228 & 0.9251978 \\
\hline
\end{tabular}


maug-cc-pVTZ

$-78.639899489803156$

$\begin{array}{lrrr}\mathrm{C} & -0.25248459 & -0.81190854 & -0.00249231 \\ \mathrm{C} & -0.20909548 & 0.68150955 & -0.00506542 \\ \mathrm{H} & 0.76612683 & -1.24913367 & 0.06539524 \\ \mathrm{H} & -0.81558862 & -1.19082096 & 0.84223612 \\ \mathrm{H} & -0.70148321 & -1.19713814 & -0.91036754 \\ \mathrm{H} & 0.03724107 & 1.20816891 & -0.90697299 \\ \mathrm{H} & -0.08175401 & 1.21480284 & 0.91733689\end{array}$

\section{CASPT2}

maug-cc-pVDZ

$-78.877807829298447$

$\begin{array}{llll}\text { C } & -0.24427308 & -0.81518338 & -0.00219939\end{array}$

C $\quad-0.17364973 \quad 0.67841938 \quad-0.00250285$

$\mathrm{H} \quad 0.76946755 \quad-1.27437821 \quad 0.06547667$

H $\quad-0.82270567 \quad-1.19344983 \quad 0.85645114$

$\mathrm{H} \quad-0.70623613 \quad-1.19930077 \quad-0.92631429$

$\mathrm{H} \quad 0.02250520 \quad 1.22655215 \quad-0.92758288$

$\mathrm{H} \quad-0.10214613 \quad 1.23282067 \quad 0.93674160$

maug-cc-pVTZ

$-78.961846193704062$

C $\quad-0.24088835 \quad-0.81136573 \quad-0.00214627$

$\begin{array}{llll}\text { C } & -0.15749429 & 0.67129619 & -0.00116451\end{array}$

H $\quad 0.75571056 \quad-1.27342106 \quad 0.06363934$

$\mathrm{H} \quad-0.81407006 \quad-1.18101482 \quad 0.84557878$

$\mathrm{H} \quad-0.70010608 \quad-1.18631275 \quad-0.91451886$

H $\quad 0.01250938 \quad 1.21516869 \quad-0.91581831$

H $\quad-0.11269915 \quad 1.22112949 \quad 0.92449982$

\section{tPBE}

maug-cc-pVDZ

$-79.019799959703974$

C $\quad-0.23809079 \quad-0.81319034 \quad-0.00247728$

$\begin{array}{llll}\text { C } & -0.14372600 & 0.67516070 & -0.00011184\end{array}$

$\mathrm{H} \quad 0.76532051 \quad-1.29176138 \quad 0.07502144$

$\mathrm{H} \quad-0.83146727 \quad-1.19153232 \quad 0.85394597$

$\mathrm{H} \quad-0.69827385 \quad-1.19764402 \quad-0.93430534$

$\mathrm{H} \quad-0.00722233 \quad 1.23643038 \quad-0.93408228$

$\mathrm{H} \quad-0.10357827 \quad 1.23801701 \quad 0.94207934$ 
maug-cc-pVTZ

$-79.042578574422834$
C $\quad-0.23409513 \quad-0.81196362$
C $\quad-0.13110686 \quad 0.67123073$
0.00152277
H $0.75864792 \quad-1.29244938$
0.00253606
H $\quad-0.74579899-1.18867224$
$-0.02911693$
H $\quad-0.77769581 \quad-1.18187341$
0.89505193
$\mathrm{H} \quad 0.14162007 \quad 1.20997730$
$-0.88048050$
H $\quad-0.26860920 \quad 1.24923062$
$-0.90154170$
0.91209838

\section{Transition state}

\section{CASSCF}

maug-cc-pVDZ

$-78.555625468087285$

$\begin{array}{cccc}\mathrm{C} & -0.59259086 & 0.00006772 & -0.18199106 \\ \mathrm{C} & 0.75555320 & 0.00000132 & 0.03945601 \\ \mathrm{H} & -1.45564099 & -0.00033404 & 1.49385645 \\ \mathrm{H} & -1.11350056 & 0.92004294 & -0.41590314 \\ \mathrm{H} & -1.11347646 & -0.91975827 & -0.41657617 \\ \mathrm{H} & 1.30173668 & -0.92372480 & 0.18279034 \\ \mathrm{H} & 1.30170498 & 0.92363973 & 0.18348577\end{array}$

maug-cc-pVTZ

$-78.578622895235554$

$\begin{array}{cccc}\mathrm{C} & -0.59002621 & 0.00008922 & -0.18167659 \\ \mathrm{C} & 0.74991032 & 0.00000915 & 0.04105577 \\ \mathrm{H} & -1.44656610 & -0.00041824 & 1.48788699 \\ \mathrm{H} & -1.10663644 & 0.91172828 & -0.41378210 \\ \mathrm{H} & -1.10664625 & -0.91139426 & -0.41437561 \\ \mathrm{H} & 1.29187095 & -0.91529984 & 0.18270542 \\ \mathrm{H} & 1.29187973 & 0.91522028 & 0.18330433\end{array}$

\section{CASPT2}

maug-cc-pVDZ

$-78.812530523691038$

$\begin{array}{cccc}\mathrm{C} & -0.57618973 & 0.00010288 & -0.21260792 \\ \mathrm{C} & 0.76469126 & 0.00001115 & 0.03867169 \\ \mathrm{H} & -1.51386972 & -0.00044896 & 1.53805854 \\ \mathrm{H} & -1.11262024 & 0.93231745 & -0.41175197 \\ \mathrm{H} & -1.11263663 & -0.93197530 & -0.41234501 \\ \mathrm{H} & 1.31719725 & -0.93429279 & 0.17224932 \\ \mathrm{H} & 1.31721380 & 0.93422017 & 0.17284355\end{array}$


maug-cc-pVTZ

$-78.895804045695044$

$\begin{array}{cccc}\mathrm{C} & -0.56582444 & 0.00010520 & -0.22134071 \\ \mathrm{C} & 0.75663282 & 0.00001095 & 0.03892382 \\ \mathrm{H} & -1.51451953 & -0.00044916 & 1.54378373 \\ \mathrm{H} & -1.09845138 & 0.92029707 & -0.40742538 \\ \mathrm{H} & -1.09846710 & -0.91995878 & -0.40801181 \\ \mathrm{H} & 1.30219994 & -0.92186175 & 0.16930049 \\ \mathrm{H} & 1.30221568 & 0.92179107 & 0.16988807\end{array}$

tPBE

maug-cc-pVDZ

$-78.950917153183440$

$\begin{array}{llll}\text { C } & -0.56417077 & 0.00012000 & -0.22984815\end{array}$

$\begin{array}{llll}\text { C } & 0.76016051 & 0.00001484 & 0.03920526\end{array}$

$\mathrm{H} \quad-1.53153879 \quad-0.00050328 \quad 1.56981636$

$\mathrm{H} \quad-1.11009335 \quad 0.93610159 \quad-0.41689485$

H $\quad-1.11012788 \quad-0.93572903 \quad-0.41745226$

H $\quad 1.31975892 \quad-0.93721708 \quad 0.16987328$

$\begin{array}{llll}\mathrm{H} & 1.31979736 & 0.93714756 & 0.17041857\end{array}$

maug-cc-pVTZ

$-78.974003730138492$

$\begin{array}{llll}\text { C } & -0.56026079 & 0.00010559 & -0.22826664\end{array}$

$\begin{array}{llll}\text { C } & 0.75407134 & 0.00000989 & 0.04029607\end{array}$

H $\quad-1.52426109 \quad-0.00044728 \quad 1.55949297$

$\mathrm{H} \quad-1.10245625 \quad 0.92656527 \quad-0.41171875$

$\mathrm{H} \quad-1.10246944 \quad-0.92622822 \quad-0.41231481$

H $\quad 1.30957486 \quad-0.92782321 \quad 0.16851510$

H $\quad 1.30958738 \quad 0.92775257 \quad 0.16911426$

R12: $\mathrm{HCN} \rightarrow \mathrm{HNC}$

nom-CPO, mod-CPO

\section{Reactants}

CASSCF

maug-cc-pVDZ

$-93.036077302129883$

$\begin{array}{llll}\text { C } & -0.50688976 & 0.00000000 & 0.00000000\end{array}$

$\begin{array}{llll}\mathrm{N} & 0.66712404 & 0.00000000 & 0.00000000\end{array}$

$\mathrm{H} \quad-1.57352829 \quad 0.00000000 \quad 0.00000000$

maug-cc-pVTZ

$-93.059512548277468$

$\begin{array}{llll}\text { C } & -0.50680349 & 0.00000000 & 0.00000000\end{array}$

$\begin{array}{llll}\mathrm{N} & 0.65658775 & 0.00000000 & 0.00000000\end{array}$

$\mathrm{H} \quad-1.56307827 \quad 0.00000000 \quad 0.00000000$ 


\section{CASPT2}

maug-cc-pVDZ

$-93.173068664375265$

$\begin{array}{llll}\text { C } & -0.50437997 & 0.00000000 & 0.00000000\end{array}$

$\begin{array}{llll}\mathrm{N} & 0.67279182 & 0.00000000 & 0.00000000\end{array}$

$\begin{array}{llll}\mathrm{H} & -1.58170585 & 0.00000000 & 0.00000000\end{array}$

maug-cc-pVTZ

$-93.251403605475247$

$\begin{array}{llll}\text { C } & -0.50439530 & 0.00000000 & 0.00000000\end{array}$

$\begin{array}{llll}\mathrm{N} & 0.65758749 & 0.00000000 & 0.00000000\end{array}$

$\begin{array}{llll}\mathrm{H} & -1.56648619 & 0.00000000 & 0.00000000\end{array}$

\section{TPBE}

maug-cc-pVDZ

$-93.289237921461449$

$\begin{array}{llll}\text { C } & -0.49966028 & 0.00000000 & 0.00000000\end{array}$

$\begin{array}{llll}\mathrm{N} & 0.67062747 & 0.00000000 & 0.00000000\end{array}$

$\mathrm{H} \quad-1.58426119 \quad 0.00000000 \quad 0.00000000$

maug-cc-pVTZ

$-93.310539168449083$

$\begin{array}{llll}\text { C } & -0.49962397 & 0.00000000 & 0.00000000\end{array}$

$\begin{array}{llll}\mathrm{N} & 0.65935610 & 0.00000000 & 0.00000000\end{array}$

$\begin{array}{llll}\mathrm{H} & -1.57302613 & 0.00000000 & 0.00000000\end{array}$

Products

CASSCF

maug-cc-pVDZ

$-93.002944545657272$

$\begin{array}{llll}\text { C } & -0.75035118 & 0.00000000 & 0.00000000\end{array}$

$\begin{array}{llll}\mathrm{N} & 0.43744794 & 0.00000000 & 0.00000000\end{array}$

$\begin{array}{llll}\mathrm{H} & 1.42812223 & 0.00000000 & 0.00000000\end{array}$

maug-cc-pVTZ

$-93.027080624721634$

$\begin{array}{llll}\text { C } & -0.74013610 & 0.00000000 & 0.00000000\end{array}$

$\begin{array}{llll}\mathrm{N} & 0.43635783 & 0.00000000 & 0.00000000\end{array}$

$\begin{array}{llll}\mathrm{H} & 1.41899726 & 0.00000000 & 0.00000000\end{array}$

\section{CASPT2}

maug-cc-pVDZ

$-93.148060155945331$

$\begin{array}{llll}\text { C } & -0.75704275 & 0.00000000 & 0.00000000\end{array}$

$\begin{array}{llll}\mathrm{N} & 0.43440526 & 0.00000000 & 0.00000000\end{array}$

$\begin{array}{llll}\mathrm{H} & 1.43785649 & 0.00000000 & 0.00000000\end{array}$

maug-cc-pVTZ

$-93.227206527561663$

$\begin{array}{llll}\text { C } & -0.74357399 & 0.00000000 & 0.00000000\end{array}$

$\begin{array}{llll}\mathrm{N} & 0.43309175 & 0.00000000 & 0.00000000\end{array}$

$\begin{array}{llll}\mathrm{H} & 1.42570124 & 0.00000000 & 0.00000000\end{array}$ 


\section{tPBE}

maug-cc-pVDZ

$-93.264830960995155$

$\begin{array}{lrrr}\mathrm{C} & -0.75690674 & 0.00000000 & 0.00000000 \\ \mathrm{~N} & 0.43085325 & 0.00000000 & 0.00000000 \\ \mathrm{H} & 1.44127249 & 0.00000000 & 0.00000000\end{array}$

maug-cc-pVTZ

$-93.286517630092376$

$\begin{array}{llll}\text { C } & -0.74613548 & 0.00000000 & 0.00000000\end{array}$

$\begin{array}{llll}\mathrm{N} & 0.42936963 & 0.00000000 & 0.00000000\end{array}$

$\mathrm{H} \quad 1.43198485 \quad 0.00000000 \quad 0.00000000$

\section{Transition state}

\section{CASSCF}

maug-cc-pVDZ

$-92.973451308092848$

$\begin{array}{llll}\text { C } & 0.06037123 & 0.60547169 & 0.00000000\end{array}$

$\begin{array}{llll}\mathrm{N} & 0.17398128 & -0.59671015 & 0.00000000\end{array}$

$\mathrm{H} \quad-1.03679551 \quad 0.09136545 \quad 0.00000000$

maug-cc-pVTZ

$-92.995313538016049$

$\begin{array}{llll}\text { C } & 0.05732259 & 0.60008009 & 0.00000000\end{array}$

$\begin{array}{llll}\mathrm{N} & 0.17176332 & -0.59187253 & 0.00000000\end{array}$

$\mathrm{H} \quad-1.03152891 \quad 0.09191943 \quad 0.00000000$

\section{CASPT2}

maug-cc-pVDZ

$-93.103571580848254$

$\begin{array}{llll}\mathrm{C} & 0.05519823 & 0.60373041 & 0.00000000\end{array}$

$\begin{array}{llll}\mathrm{N} & 0.17855396 & -0.59992616 & 0.00000000\end{array}$

$\mathrm{H} \quad-1.03619519 \quad 0.09632274 \quad 0.00000000$

maug-cc-pVTZ

$-93.179864503684072$

$\begin{array}{llll}\text { C } & 0.05100804 & 0.59605793 & 0.00000000\end{array}$

$\begin{array}{llll}\mathrm{N} & 0.17420968 & -0.59229323 & 0.00000000\end{array}$

$\mathrm{H} \quad-1.02766072 \quad 0.09636230 \quad 0.00000000$

tPBE

maug-cc-pVDZ

$-93.216900983684951$

$\begin{array}{llll}\text { C } & 0.06478508 & 0.60760365 & 0.00000000\end{array}$

$\begin{array}{llll}\mathrm{N} & 0.16817014 & -0.59344962 & 0.00000000\end{array}$

$\mathrm{H} \quad-1.03539822 \quad 0.08597296 \quad 0.00000000$ 
maug-cc-pVTZ

$-93.236936433314028$

$\begin{array}{llll}\text { C } & 0.06261065 & 0.60253190 & 0.00000000\end{array}$

$\begin{array}{llll}\mathrm{N} & 0.16362111 & -0.58720269 & 0.00000000\end{array}$

H $\quad-1.02867476 \quad 0.08479779 \quad 0.00000000$ 